\title{
An Adaptive Harmonic Polynomial Cell Method with Immersed Boundaries: Accuracy, Stability and Applications
}

Tong, Chao; Shao, Yanlin; Bingham, Harry B.; Hanssen, FinnChristian W.

Published in:

International Journal for Numerical Methods in Engineering

Link to article, DOI:

10.1002/nme.6648

Publication date:

2021

Document Version

Peer reviewed version

Link back to DTU Orbit

Citation (APA):

Tong, C., Shao, Y., Bingham, H. B., \& Hanssen, FC. W. (2021). An Adaptive Harmonic Polynomial Cell Method with Immersed Boundaries: Accuracy, Stability and Applications. International Journal for Numerical Methods in Engineering, 122, 2945-2980. https://doi.org/10.1002/nme.6648

\section{General rights}

Copyright and moral rights for the publications made accessible in the public portal are retained by the authors and/or other copyright owners and it is a condition of accessing publications that users recognise and abide by the legal requirements associated with these rights.

- Users may download and print one copy of any publication from the public portal for the purpose of private study or research.

- You may not further distribute the material or use it for any profit-making activity or commercial gain

- You may freely distribute the URL identifying the publication in the public portal 


\title{
An Adaptive Harmonic Polynomial Cell Method with Immersed Boundaries: Accuracy, Stability and Applications
}

\author{
Chao Tong $^{1 *}$, Yanlin Shao ${ }^{1,2 \dagger}$, Harry B. Bingham ${ }^{1}$, Finn-Christian W. Hanssen ${ }^{3}$ \\ 1: Department of Mechanical Engineering, Technical University of Denmark, 2800, Lyngby, Denmark \\ 2: College of Shipbuilding Engineering, Harbin Engineering University, 150001, Harbin, China \\ 3: Centre for Autonomous Marine Operations and Systems (AMOS), Department of Marine Technology, \\ N-7491, Trondheim, Norway
}

\begin{abstract}
We present a 2D high-order and easily accessible immersed-boundary adaptive Harmonic Polynomial Cell (IB-AHPC) method to solve fully-nonlinear wave-structure interaction problems in marine hydrodynamics using potential-flow theory. To reduce the total number of cells without losing accuracy, adaptive quad-tree cell refinements are employed close to the free-surface and structure boundaries. The present method is simpler to implement than the existing IB-HPC alternatives, in that it uses standard square cells both in the fluid domain and at the boundaries, thus without having to use the more complex and expensive overlapping grids or irregular cells. The spurious force oscillations on moving structures, which is a known issue for immersed boundary methods (IBMs), are eliminated in this study by solving a separate boundary value problem (BVP) for a Lagrangian acceleration potential. We also demonstrate that solving a similar BVP for the corresponding Eulerian acceleration potential is far less satisfactory due to the involved second derivatives of the velocity potential in the body-boundary condition, which are very difficult to calculate accurately in an IBM-based approach. In addition, we present, perhaps for the first time since the HPC method was developed, a linear matrix-based stability analysis for the time-domain IB-AHPC method. The stability analysis is also used in this study as a general guide to design robust and stable numerical algorithms, in particular related to the treatment of boundary conditions close to the intersection between a Dirichlet and a Neumann boundary, which is essential in time-domain wave-structure interaction analyses using IBMs. We confirm theoretically through the stability analysis that square cells have the best stability properties. The present method has been verified and validated satisfactorily by various cases in marine hydrodynamics, including a moving structure in an infinite fluid, fully-nonlinear wave generation and propagation, and fully-nonlinear diffraction and radiation of a ship cross section.
\end{abstract}

Keywords: Harmonic Polynomial Cell method; Adaptive quad-tree cells; Potential flow; Immersed boundary method; Accuracy and stability analysis; Fully-nonlinear wave-body interaction

\section{Introduction}

Analysis of ships, and coastal and offshore structures, inevitably involves the modeling of water waves and their interaction with the structures. The natural physics of ocean flows can be well described by the Navier-Stokes (NS) equations with proper turbulence modeling [1, 2], using Computational Fluid Dynamics (CFD) methods. CFD can be expected to accurately capture the complicated flow details at small scales due to wave-structure interaction, in particular for physical processes such as wave breaking, turbulence, air entrapment and water jets. In the last decades,

*Email address: ctong@mek.dtu.dk

$\dagger$ Email address: yshao@mek.dtu.dk

This article has been accepted for publication and undergone full peer review but has not been through the copyediting, typesetting, pagination and proofreading process which may lead to differences between this version and the Version of Record. Please cite this article as doi: $10.1002 /$ nme.6648 
43 method by Hague and Swan [28].

In contrast to the BEMs, field or volume methods discretize the entire fluid domain. Li and

many CFD solvers have been developed and become widely used for various hydrodynamic studies. Some of the CFD solvers are preferred in the scientific and engineering communities, such as opensource tools like OpenFOAM $\mathbb{R}[3,4,5]$ and REEF3D::CFD [6, 7], and commercial softwares like STAR-CCM+ [8] and ANSYS-FLUENT [9]. Highly accurate NS solvers have also been developed in recent years with success. Among others, a new staggered space-time discontinuous Galerkin method (DGM) has been proposed by Tavelli and Dumbser [10] to solve the NS equations, which was reported to reach arbitrarily high-order accuracy both in time and space. More information on families of staggered DGMs for solving the NS equations can be found in e.g. [11, 12, 13]. Although a closer investigation of complex flows for wave-structure interaction can be achieved by these Navier-Stokes solvers, fine mesh resolution in the computational domain is required to fully capture the flow field. This makes them costly in terms of both CPU time and memory, which is the main bottleneck and challenge of applying CFD method for problems involving large-scale wave domains.

For large-volume marine structures, viscous effects are generally of secondary importance, and thus can be accounted for empirically as an engineering practice. It is therefore convenient and ideal to use potential-flow theory to predict the loading on, and response of, these structures $[14,15,16]$. The conventional approach to model waves and wave-structure interaction within the context of potential flow is the boundary element method (BEM) [17, 18, 19, 20, 21]. A high-order BEM was proposed in [18] to solve various strongly nonlinear wave problems like wave shoaling, run-up and plunging breakers. Among others, Ferrant et al. [19] and Bai and Eatock Taylor [22] applied BEMs to solve fully-nonlinear wave-structure interactions with success. Unlike other weakly-nonlinear approaches based on perturbation schemes $[23,20]$, the exact fully-nonlinear free-surface boundary conditions are integrated in the time domain to update the wave profile and velocity potential on the instantaneous free surface. With $N$ the number of unknowns on the boundaries of the computational domain, a classical BEM takes $O\left(N^{2}\right)$ operations to build up the influence coefficients for the discretized boundary integral equations. Solving the resulting dense-matrix equations takes CPU time proportional to $O\left(N^{3}\right)$ for a direct method such as Gaussian-elimination or LU-factorization, and $O\left(N^{2}\right)$ for an iterative solver. See the discussions in [24, 25]. This may lead to excessive CPU time for conventional BEMs, particularly for large-scale problems. On the other hand, thanks to we enhancement of BEMs, the operation complexity is notably reported to be reduced to $O(N)$ by using the fast multipole accelerated (FMA) method and $O(N \log N)$ with pre-corrected FFT (pFFT) techniques $[26,27]$. Another challenge for the application of BEMs is the corner problem at the wave-body intersection. Extra attention needs to be paid to Dirichlet-Neumann-boundary corners where the normal directions of the boundaries are discontinuous, otherwise it may lead to unstable solutions [28, 29]. This kind of problem can be effectively solved by either the double-node collocation techniques proposed by Grilli and Svendsen [29] and Tanizawa [30], or the multiple flux Fleming [31] originally applied a finite difference method (FDM) with a low-order multigrid technique to solve the Laplace equation for the fully-nonlinear potential-flow problems. Bingham and Zhang [32] further improved this model with a high-order finite difference scheme. This model was then extended to three dimensions, namely in the open-source code OceanWave3D by Engsig-Karup et al. [33] to study 3D nonlinear water waves with a flexible-order finite difference approximation. OceanWave3D was later enhanced with a GPU-based parallelization to increase the computational efficiency by Engsig-Karup et al. [34]. The finite element method (FEM) has also been successfully applied to study fully-nonlinear potential-flow problems. See for instance [35, 36, 37, 38]. A novel computational procedure to solve potential-flow problems is called the scaled boundary finite element method (SBFEM). This method combines the advantages both from the FEM, that no fundamental 
solutions are required, and from the BEM, that the spatial dimension is reduced by one [39, 40,41, 42]. A coupled FEM and BEM can also be implemented to study wave-body interactions, where the FEM is used away from the body while the BEM is used in vicinity of the body (See, e.g. [43, 36]). The combination is based on consideration of the efficiency of FEM and BEM in computation and mesh generation, respectively.

Toward high accuracy and efficiency field methods for solving fully-nonlinear potential-flow problems, a novel method, namely the Harmonic Polynomial Cell (HPC) method was proposed by Shao and Faltinsen [24] first in two dimensions (2D) and later extended to three dimensions (3D) by Shao and Faltinsen [44]. In recent years, many researchers have shown great interest in the application and further development of the HPC method. The local and global accuracy features of the 2D HPC method were investigated in depth by Ma et al. [45], who concluded that the local errors can be minimized by applying square-shaped cells. Bardazzi et al. [46] generalized the conventional 2D HPC method to solve the Poisson equation with success. The HPC solver combined with a viscous-flow solver through a domain-decomposition scheme was proposed by Hanssen [47] and Fredriksen et al. [48]. By doing so, in the region where the flow changes rapidly and viscosity cannot be neglected, the Navier-Stokes equations are applied, while in the bulk of the fluid the HPC method is used to dramatically reduce the computational time. The original HPC method, both in $2 \mathrm{D}$ and 3D, was developed based on boundary-fitted meshes, which makes it cumbersome for complex boundaries especially in 3D problems. Therefore, alternative techniques have been proposed to improve and enhance this method.

Hanssen et al. [49] and Zhu et al. [50] were the first to introduce the concept of the immersed boundary method (IBM) to the 2D HPC method. However, it was reported that the use of IBM can introduce spurious pressure oscillations for moving structures. To overcome this disadvantage, Hanssen et al. [51] and Hanssen [47] resorted to an overlapping-grid strategy, where a local grid attached to the structure overlaps with another global background grid. The local grid can be finer than the global grid to increase the accuracy of the solution. Since there is no relative motion between the body and the local grid, variation in the truncation errors at a moving surface introduced by an ordinary IBM are minimized, which also minimizes the spurious pressure oscillations. Very recently, hobaux and Benoit [52] also applied a similar overlapping-grid strategy in their development of a fully-nonlinear numerical wave tank based on the HPC method. Liang et al. [53] also applied the overlapping grids in a 3D HPC method to study liquid sloshing in an upright circular tank. Tong et al. [54] used the method developed in Hanssen et al. [51] to systematically study the generation and interaction of solitary waves in a fully-nonlinear numerical wave tank. However, applying this overlapping-grid strategy involves two-way information exchange between the local and the global grids, which means that the size and topology of the global coefficient matrix changes with time. This is a disadvantage compared to using a boundary-fitted approach since the global coefficient matrix must be re-constructed at each time step. At the same time, for deep water and large wave domains, from an accuracy point of view, if square cells are adopted, the unknowns will dramatically increase, which increases the computational effort. Furthermore, as observed in Robaux and Benoit [52], instabilities on the free surface in the overlapping zone may occur due to reciprocal interpolation between two grid systems. To overcome this, Robaux and Benoit [52] proposed to use a free-surface relaxation between the local and global grids.

Another interesting development in the 2D HPC method was made recently by Wang et al. [25] and Shen et al. [55], where irregular cells close to the boundaries are constructed through a local approximation based on least-square fitting of harmonic polynomials. A similar idea has been discussed earlier by Shao and Faltinsen [44] in their formulation of the original 3D HPC method. It also has similarities with the weighted-least square (WLS) strategies applied in the FDM. An 


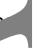

121

122

123

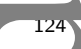

advantage of using irregular cells is that arbitrary order of polynomials could be included in the local approximations. With a proper free-surface tracking method, Wang et al. [25] have been able to deal with problems involving complex boundaries, i.e. plunging breakers, sloshing and water entry. However, a local irregular cell has to be formed for each boundary point at each time step in fully-nonlinear wave-structure interaction analysis, where the free surface and structure surfaces move or deform. Since a number of stencil points, typically much larger than that of a standard cell, are needed to form a solvable WLS problem, it can potentially increase the complexity and the computational cost of the method [55].

In the present study, we propose a new immersed-boundary HPC method, which avoids having to use the overlapping-grid strategy $[51,47,52]$, or the irregular cells $[25,55]$ to handle complex geometries. As will be shown in later sections, the method has been satisfactorily verified and validated by several cases including: a circular cylinder moving in an infinite fluid where an analytical solution exists, fully-nonlinear wave generation and propagation, and fully-nonlinear diffraction and radiation of a ship cross section. The agreement with existing theoretical and experimental references demonstrate the satisfactory accuracy, stability and feasibility of the method.

The features and novelty of the present work are summarized as follows: Standard $4^{\text {th }}$-order square-shaped cells are consistently used both in the fluid domain and at the fluid boundaries so that no additional effort is needed to construct the local cells at each boundary node and each time step, since the interpolation coefficients of square cells are constant and easily scaled. Secondly, the cells are adaptively refined at the boundaries, either automatically or controlled by the user, to increase the accuracy where needed. This greatly reduces the number of unknowns to achieve a required accuracy; Thirdly, we show that the spurious force oscillations reported in the literature $[49,56,47]$ for the IBM can be eliminated by solving a separate boundary value problem (BVP) for the Lagrangian acceleration potential. Another similar and widely used approach in fully-nonlinear potential-flow analysis, which solves the Eulerian acceleration potential, can reduce the force oscillations only if the grid close to the solid structure is fine enough. However, it does not eliminate the spurious force oscillations satisfactorily with an affordable cell resolution. This is related to the required second derivatives of the velocity potential at the structure surface in the associated BVP; Last but not least, we present, perhaps for the first time in the literature, a linear matrix-based stability analysis for the HPC method, which serves as a useful guide in the design of our numerical algorithms: including understanding the effect of the grid stretching and the proper treatment of Neumann boundaries to ensure the stability of the method.

The reminder of this paper is organized as follows. In $\S 2$, the formulation of the potential-flow problem with fully-nonlinear boundary conditions is presented. $\S 3$ outlines the basic theory about the HPC method, the IBM for free surfaces and body boundaries, and the Lagrangian acceleration potential method. In $\S 4$ the grid system and the procedure of domain discretization are presented in detail. $\S 5$ provides the validation of accuracy and spatial convergence for the numerical model, and a matrix-based stability analysis is also presented in this section. Three applications of this numerical model are given in $\S 6$. In the end, $\S 7$ summarizes the present work.

\section{$2 \quad$ Fully nonlinear wave-structure interaction model}

\subsection{Governing equation and boundary conditions}

A 2D Cartesian coordinate system $O x z$ is defined with its origin fixed at the middle of a numerical wave tank, the $O x$ axis coinciding with the undisturbed free surface and $O z$ axis orienting positively 
upward, as shown in Fig. 1. By applying potential-flow theory, the fluid is assumed to be ideal, without viscosity. The flow is considered as irrotational and the fluid is incompressible. The fluid motion in the domain $\Omega$ can then be described by a velocity potential $\varphi(x, z)$, which satisfies the Laplace equation:

$$
\frac{\partial^{2} \varphi}{\partial x^{2}}+\frac{\partial^{2} \varphi}{\partial z^{2}}=0, \text { in } \Omega_{\text {fluid }}
$$

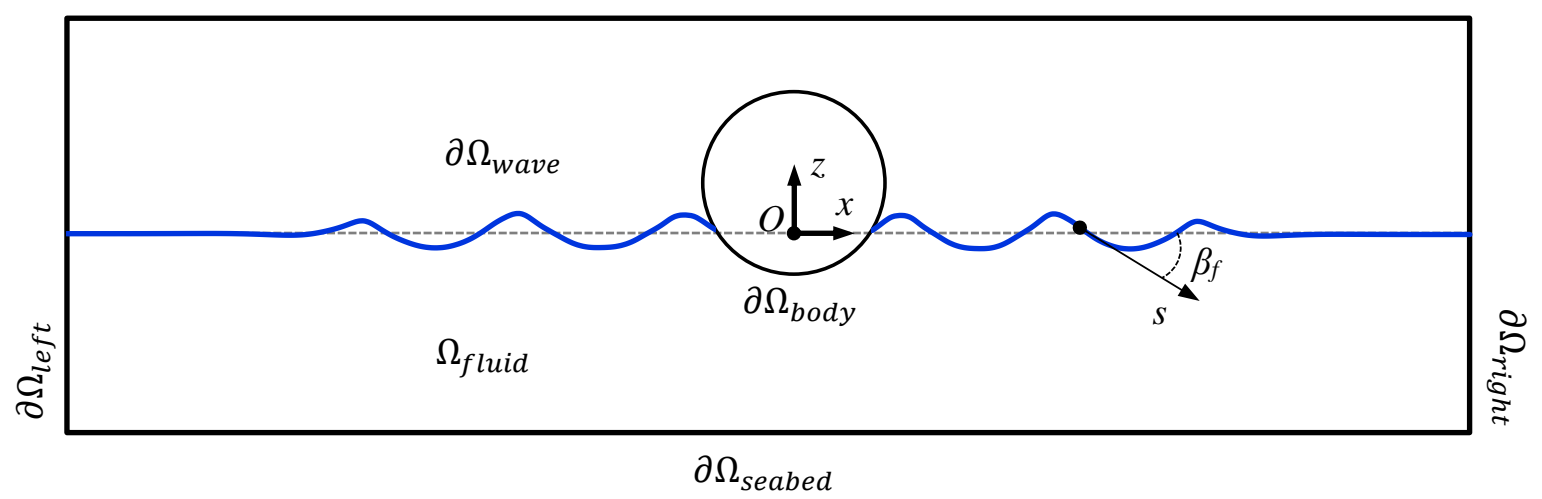

Figure 1: Sketch of wave-body interaction model in a numerical wave tank.

Along $\partial \Omega_{\text {wave }}$, the coordinates of a point on the free surface are denoted as $(x(t), \eta(x, t))$ in the inertial reference frame. The free-surface boundary conditions in the inertial reference frame are expressed by the Lagrangian time derivative $\delta / \delta t=\partial / \partial t+\boldsymbol{v} \cdot \nabla$ as:

$$
\begin{aligned}
& \begin{cases}\frac{\delta x}{\delta t}=\frac{\partial \varphi}{\partial x}+(\boldsymbol{v}-\nabla \varphi) \cdot \nabla x, & \text { on } \partial \Omega_{w a v e}, \\
\frac{\delta \eta}{\delta t}=\frac{\partial \varphi}{\partial z}+(\boldsymbol{v}-\nabla \varphi) \cdot \nabla \eta-\nu(x) \eta, & \\
\frac{\delta \varphi}{\delta t}=-\frac{1}{2}|\nabla \varphi|^{2}-g \eta+\boldsymbol{v} \cdot \nabla \varphi-\nu(x) \varphi, & \text { on } \partial \Omega_{\text {wave }},\end{cases}
\end{aligned}
$$

where $g$ is the gravitational acceleration, $\nabla x=(1,0)$ and $\nabla \eta=(\partial \eta / \partial x, 0)$. Here $\boldsymbol{v}=0$ corresponds to an Eulerian description, while $\boldsymbol{v}=\nabla \varphi$ corresponds to the fully Lagrangian description and $\boldsymbol{v}=(0, \partial \eta / \partial t)$ gives a semi-Lagrangian description. $\nu(x)$ is a damping coefficient which is only active in the wave-absorbing zone and is zero elsewhere. In Eq. (3), $\partial \varphi / \partial t=-\frac{1}{2}|\nabla \varphi|^{2}-g \eta$ is derived from Bernoulli's equation where the water pressure on the free surface is equal to the constant atmospheric pressure. The fully-nonlinear free surface conditions are integrated forward in time using the explicit $4^{\text {th }}$-order Runge-Kutta scheme.

The fluid domain is enclosed by either Dirichlet boundaries $\Gamma_{D}$ or Neumann boundaries $\Gamma_{N}$. In order to solve the Laplace equation, Dirichlet and Neumann boundary conditions are needed and given as:

$$
\begin{gathered}
\varphi(x, z)=\varphi_{D}, \quad \text { on } \Gamma_{\mathrm{D}} \\
\frac{\partial \varphi(x, z)}{\partial n}=\nabla \varphi \cdot \mathbf{n}, \quad \text { on } \Gamma_{\mathrm{N}}
\end{gathered}
$$


$\sqrt{1}$

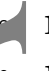

179

180

181 102

where $\mathbf{n}$ denotes the unit normal vector on Neumann boundaries pointing into the fluid domain. On the Neumann boundaries, the fluid cannot penetrate the body surface so that the right-hand side of Eq. (5) equals the normal velocity of the body boundary. For a numerical wave tank, Dirichlet boundary conditions are imposed on the free surface. The right-hand side of Eq. (4) comes from the time integration of the dynamic boundary condition on the free surface in Eq. (3). For a wave-body interaction problem, a mixed Dirichlet-Neumann boundary value problem (BVP) will be solved for the velocity potential at each time step.

\subsection{Wave-body surface intersection problem}

The intersection of the wave-body surface can cause numerical problems due to the discontinuity of flux, if one does not properly take care of this. Proper treatment of the intersection point is not only of significant importance to the global accuracy and stability of the numerical solution, but also critical to the prediction of wave run-up along the body surface and the loads on floating structures. To make sure that the intersection point remains on both the body surface and the free surface during the time integration, Liu et al. [57] provided a velocity formula at the intersection point to accomplish this goal. Tanizawa [30] used a double-node technique to deal with the discontinuity of flux at the intersection point for the BEM. Hague and Swan [28] proposed a multiple flux BEM to replace the double-node collocation with a single node to be placed at the intersection point. This method was proven to be more accurate than the double-node techniques for wave propagation and wave-body interactions.

In this paper, the velocity of the wave-body intersection point on the free surface is transformed from a Cartesian coordinate system into a curvilinear coordinate system:

$$
\left\{\begin{array}{l}
\frac{\partial \varphi_{f}}{\partial x}=\frac{\partial \varphi_{f}}{\partial s} \cos \beta_{f}-\frac{\partial \varphi_{f}}{\partial n} \sin \beta_{f}, \\
\frac{\partial \varphi_{f}}{\partial z}=\frac{\partial \varphi_{f}}{\partial s} \sin \beta_{f}+\frac{\partial \varphi_{f}}{\partial n} \cos \beta_{f}
\end{array}\right.
$$

183 where $\beta_{f} \in\left(-\frac{\pi}{2}, \frac{\pi}{2}\right)$ is the angle between the $s$ - and the $x$-axis, defined clockwise positive. As shown in Fig. 1, the $s$-axis coincides with the tangential direction to the free surface pointing in the positive direction of the $x$-axis, and $\beta_{f}$ can be determined by

$$
\beta_{f}=\arctan \left(-\frac{\partial \eta}{\partial x}\right)
$$

Here $\partial \eta / \partial x$ is the slope of the free surface, which will be obtained in this study by a cubic spline fitting. In order to deal with the flux discontinuity at the intersection point, we will follow the approach by Grilli and Svendsen [29] to modify the tangential velocity $\partial \varphi_{f} / \partial s$ in Eq. (6) as:

$$
\frac{\partial \varphi_{f}}{\partial s}=\frac{\partial \varphi_{b}}{\partial n} \cdot \frac{1}{\sin \left(\beta_{f}-\beta_{b}\right)}-\frac{\partial \varphi_{f}}{\partial n} \cdot \frac{1}{\tan \left(\beta_{f}-\beta_{b}\right)},
$$

where $\partial \varphi_{b} / \partial n$ and $\partial \varphi_{f} / \partial n$ are the normal velocities at the intersection point to the body surface and the free surface, respectively. Note that the normal vector of either the body or the free surface is pointing outside of the body or the fluid domain. $\beta_{b}$ is defined as the angle between the normal vector to the body surface and the $z$-axis. If the angle is defined positive in a clockwise direction, then $\beta_{b}=\pi / 2$ at left tank wall and $\beta_{b}=3 \pi / 2$ at right tank wall. 

into Eq. (9):

$$
\left[\begin{array}{ccc}
p_{1}\left(\xi_{1}, \zeta_{1}\right) & \ldots & p_{8}\left(\xi_{1}, \zeta_{1}\right) \\
\vdots & p_{j}\left(\xi_{i}, \zeta_{i}\right) & \vdots \\
p_{1}\left(\xi_{8}, \zeta_{8}\right) & \cdots & p_{8}\left(\xi_{8}, \zeta_{8}\right)
\end{array}\right] \cdot\left[\begin{array}{c}
b_{1} \\
\vdots \\
b_{j} \\
\vdots \\
b_{8}
\end{array}\right]=\left[\begin{array}{c}
\varphi_{1} \\
\vdots \\
\varphi_{i} \\
\vdots \\
\varphi_{8}
\end{array}\right] \Longrightarrow \mathcal{P} \mathbf{b}=\boldsymbol{\varphi}
$$

the unkonwn coefficients $b_{j}(j=1,2, \cdots, 8)$ in vector $\mathbf{b}$ can be resolved as:

$$
\mathbf{b}=\mathcal{C} \varphi
$$

where matrix $\mathcal{C}$ (with elements $c_{j, i}(j, i=1,2, \cdots, 8)$ ) is the inverse of the matrix $\mathcal{P}$, whose elements are $p_{i, j}=p_{j}\left(\xi_{i}, \zeta_{i}\right),(j, i=1,2, \cdots, 8)$. Note that both matrices $\mathcal{P}$ and $\mathcal{C}$ are only dependent on the cell geometry. Therefore, they are constant if the cell does not change. Substituting Eq. (11) into the interpolation equation (9) yields

$$
\varphi(\xi, \zeta)=\mathbf{p}(\xi, \zeta) \cdot \mathcal{C} \varphi
$$

Eq. (12) indicates that the potential at any point within a cell can be approximated by the linear combination of the velocity potential on the eight border nodes of the cell. One could make an analogy of this approximation to a finite difference or node-based finite element approximation. The connectivity equation for the velocity potential in the fluid domain is obtained by inserting the local coordinates of the central point $i=9$ into Eq. (12):

$$
\varphi_{9}=\varphi(0,0)=\mathbf{c} \cdot \varphi \text {. }
$$




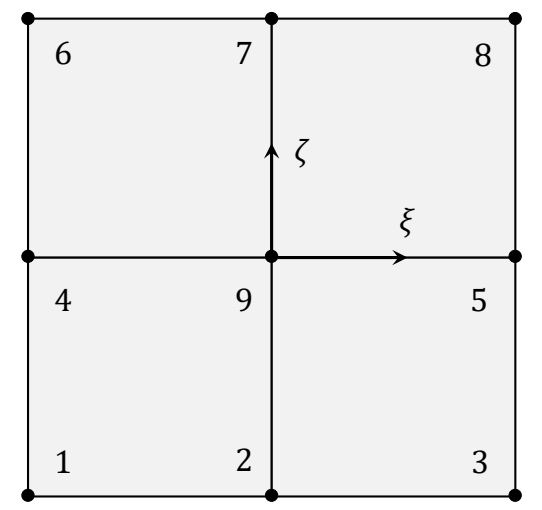

Figure 2: Sketch of a cell with local indices and coordinate system.

Table 1: List of harmonic polynomials categorized into real and imaginary part of $(\xi+\mathrm{i} \zeta)^{n}$.

\begin{tabular}{l|l|l}
$n$ & $\mathfrak{u}(\xi, \zeta)$ & $\mathfrak{v}(\xi, \zeta)$ \\
\hline 0 & $p_{1}(\xi, \zeta)=1$ & - \\
1 & $p_{2}(\xi, \zeta)=\xi$ & $p_{3}(\xi, \zeta)=\zeta$ \\
2 & $p_{4}(\xi, \zeta)=\xi^{2}-\zeta^{2}$ & $p_{5}(\xi, \zeta)=\xi \zeta$ \\
3 & $p_{6}(\xi, \zeta)=\xi^{3}-3 \xi \zeta^{2}$ & $p_{7}(\xi, \zeta)=3 \xi^{2} \zeta-\zeta^{3}$ \\
4 & $p_{8}(\xi, \zeta)=\xi^{4}-6 \xi^{2} \zeta^{2}+\zeta^{4}$ & -
\end{tabular}

Here $\mathbf{c},\left(c_{1, i}, i=1,2, \cdots, 8\right)$ is the first row of matrix $\mathcal{C}$. As Eq. (12) serves as Dirichlet boundary conditions, and Eq. (13) is applied for inner fluid nodes, then on the solid boundaries, the normal derivative of the velocity potential involved in the Neumann boundary condition is obtained by directly taking the normal derivative of the harmonic polynomials:

$$
\frac{\partial \varphi}{\partial n}(\xi, \zeta)=\mathbf{q}(\xi, \zeta) \cdot \mathcal{C} \boldsymbol{\varphi}
$$

225 where the element of the vector $\mathbf{q}(\xi, \zeta)$ is defined as $q_{j}(\xi, \zeta)=\nabla p_{j}(\xi, \zeta) \cdot \mathbf{n}(\xi, \zeta),(j=1,2, \ldots, 8)$. Here, $\mathbf{n}(\xi, \zeta)$ is the normal vector on the boundary. Since the harmonic polynomials automatically satisfy the Laplace equation, imposing the Laplace equation numerically becomes unnecessary in this case. This feature is a natural advantage for the HPC method compared to other potential-flow field solvers. In theory, the accuracy of the HPC method can reach arbitrary orders as long as highorder harmonic polynomials are utilized. In this case, the cell can be changed into irregular shapes containing the necessary number of nodes to reach a desired accuracy [44, 25]. Establishing the adequate equations for all active nodes in the computational domain, the linear algebraic equation system is established as $\mathcal{A} \boldsymbol{\varphi}=\mathbf{e}$, with $\mathcal{A}$ the coefficient matrix containing at most nine entries in each row, $\varphi$ the unknown velocity-potential vector and e the boundary-condition vector.

\subsection{Free surface and structure geometry modelled as immersed bound- aries}

As generation of body-fitted grids could be cumbersome for structures with complex and/or moving geometry, the immersed boundary method (IBM) was proposed and implemented to make it 

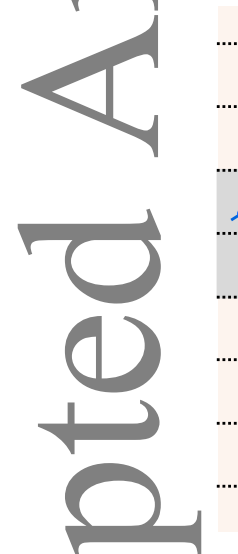

easy to deal with arbitrary geometries in regular grids. Hanssen et al. [49] first introduced the IBM technique to the HPC method for a moving rigid body in an infinite fluid domain. Later on, Hanssen et al. [51] enhanced this idea to the free surface in a numerical wave tank and proved that this method works well for various wave propagation problems, such as solitary waves in shallow water, focused waves and periodic waves in shallow and deep water. Moreover, Hanssen [47] successfully treated both the free surface and the body surface as immersed boundaries at the same time to study fully-nonlinear wave-body interaction. In the work by Hanssen et al. [49], spurious force oscillations were observed when the structure moves relative to the fixed grid. The main reason for these spurious oscillations was that the time derivative of the velocity potential, needed to compute the pressure in Bernoulli's equation, was estimated using backward finite difference. By instead solving a separate BVP for the time derivative of the velocity potential in [47], the spurious pressure oscillations are avoided. Hanssen et al. [51] and Hanssen [47] introduced overlapping, structured grids following the motion of the moving bodies. This was mainly done in order to refine the solution locally, but it also eased the formulation of the body-boundary condition in the BVP for the time derivative of the velocity potential. An IBM to refine the grid locally without using overlapping grids, while avoiding spurious pressure oscillations, will be discussed in detail in the following sections.

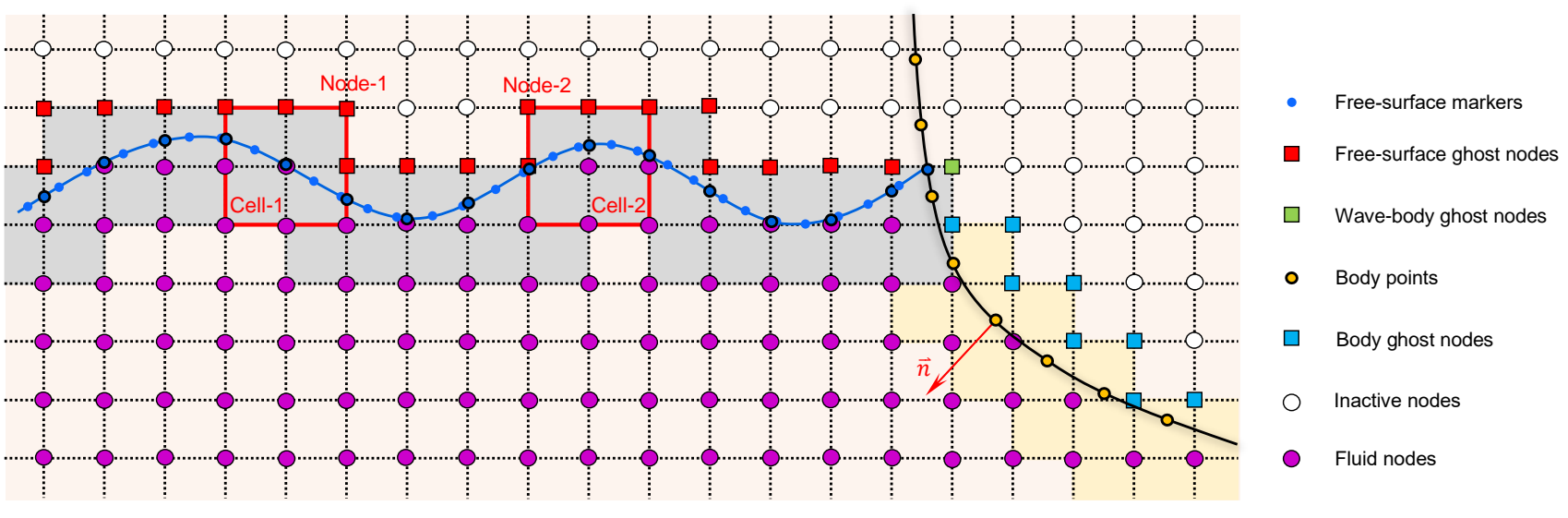

Figure 3: Immersed boundary method for free surface and body geometry. The free surface in a structured Cartesian grid is tracked by markers, and the body surface is represented by body points. The cells shaded in gray are used to impose free-surface conditions, while the cells shaded in yellow are used to impose Neumann boundary conditions.

Fig. 3 shows the free surface and body boundary modelled as immersed boundaries. The free surface is discretized with a set of wave markers (blue circles with or without a black edge), and the body is represented by a set of discrete points (yellow circles with a black edge). All the node types are defined in Fig. 3. For the interior fluid nodes, the connectivity equation (13) will be applied to ensure the continuity of the velocity potential and its time derivative across the fluid domain.

To impose Dirichlet boundary conditions on the free surface, the velocity potential on the wave markers will be interpolated using a proper cell, which always contains ghost nodes in its border nodes. The selected cells for the wave markers will be referred as free-surface cells in this paper. Two different types of wave markers, namely the semi-Lagrangian and fully Lagrangian markers, are defined. Fully Lagrangian markers are only used close to the body surface, while semi-Lagrangian wave markers are used elsewhere on the free surface. The semi-Lagrangian wave markers (blue circles with a black edge excluding the one on the body boundary) are only allowed to move along vertical 

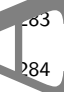

285

286

287)

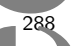

280

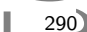

( 290

292

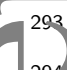

29

295

297

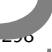

300

301

grid lines. They are always within certain free-surface cells, where the markers are located on the upper half of the vertical central lines of the cells. The fully Lagrangian wave markers (blue circles without a black edge) are free to move horizontally and vertically, following the material velocity of the water particles. A cell will be associated to a Lagrangian wave marker, if the following two conditions are met: (1) The marker is located at the upper part of the cell; (2) Among other neighbor cells, the marker is closest to the vertical central line of the selected cell. Then this marker is defined to be an active marker. This principle of selecting the free-surface cells is inspired by the observation in [45] that the interpolation accuracy along the middle lines of the harmonic polynomial cells is the best. Referring to Fig. 2, the nodes with local index-7 in a free-surface cell will be denoted as a free-surface ghost node. If we denote the coordinates of an active marker as $\left(\xi_{m}, \zeta_{m}\right)$ in the local cell coordinate system, the Dirichlet free-surface boundary condition is imposed as

$$
\mathbf{p}\left(\xi_{m}, \zeta_{m}\right) \cdot \mathcal{C} \boldsymbol{\varphi}=\varphi_{m}
$$

where $\varphi_{m}$ is the velocity potential on the wave maker. Here, both the coordinates $\left(\xi_{m}, \zeta_{m}\right)$ and the velocity potential $\varphi_{m}$ of a wave maker can be directly obtained by time-integration of the free-surface conditions in Eq. (2) and (3), respectively. Meanwhile, the matrix $\mathcal{C}$ is determined once the geometry of the cell is fixed and $\mathbf{p}\left(\xi_{m}, \zeta_{m}\right)$ can also be calculated if the coordinates $\left(\xi_{m}, \zeta_{m}\right)$ of a wave maker are known, so that Eq. (15) can be accounted for in the global matrix system based only on the unknown velocity potential $\varphi$ on the border nodes of a cell to be resolved. In general, the number of active wave markers should be the same as that of the free-surface ghost nodes. If there is more than one ghost node on a cell border, called additional free-surface ghost nodes, see Node-1 and Node-2 in Cell-1 and Cell-2 respectively in Fig. 3 for example, then extra wave makers will be created on the free surface by interpolating the wave elevation and velocity potential from the other wave makers. A cubic B-spline interpolation is used for this purpose. Generally, this extra wave maker is below the additional free-surface ghost node and coincides with the intersection point between the free surface and the vertical grid line where the additional free-surface ghost node is located. In summary, the number of Dirichlet free-surface boundary condition equations is the same as that of the ghost nodes above the free surface.

As for the body boundary condition, body ghost nodes and body cells have to be identified first. If a grid node is inside the body, and at least one of its eight surrounding grid nodes are outside the body, it will be labeled as a body ghost node. A body cell should contain part of the body boundary, and its border nodes should not include any inactive nodes. Similar to what we have done for the wave markers, the Neumann boundary condition at a body point (denoted as body points in Fig. 3) will be imposed using the interpolation from the corresponding body cell. The total number of body ghost nodes should be the same as that of the active body points. Therefore, for each body ghost node, a body point closest to this ghost node will be created on the body surface, and its coordinates and normal direction are obtained by using a cubic B-spline interpolation. In practice, we always use a high resolution of body points to describe the body geometry to minimize the errors introduced by interpolation. If we denote the coordinates of a body point as $\left(\xi_{b}, \zeta_{b}\right)$ in the local cell coordinate system, and the corresponding rigid-body velocity at this point is $\mathbf{V}$, then the body boundary condition is imposed as

$$
\mathbf{q}\left(\xi_{b}, \zeta_{b}\right) \cdot \mathcal{C} \boldsymbol{\varphi}=\frac{\partial \varphi}{\partial n}\left(\xi_{b}, \zeta_{b}\right)=\mathbf{V} \cdot \mathbf{n}
$$

where $\mathbf{n}$ is the unit normal vector at the corresponding body point pointing into fluid.

As seen in Fig. 3, there is a special ghost node marked in green with a black edge, called the wave-body ghost node. For this kind of node, since it is associated with the intersection between 


\subsection{The Lagrangian acceleration potential method}

The instantaneous pressure on the body surface can be computed from Bernoulli's equation:

$$
P=-\rho\left(\varphi_{t}+\frac{1}{2}|\nabla \varphi|^{2}+g z\right)
$$

Integrating the pressure over the wetted body surface yields the fluid forces $\mathbf{F}_{\text {hydro }}$ and moments $\mathbf{M}_{\text {hydro }}$ on the body:

$$
\mathbf{F}_{\text {hydro }}=-\int_{\Gamma_{S}} P \mathbf{n} d s, \quad \mathbf{M}_{\text {hydro }}=-\int_{\Gamma_{S}} P \mathbf{r} \times \mathbf{n} d s .
$$

where $\mathbf{n}$ is the normal vector pointing into the fluid. In order to get $\mathbf{F}_{\text {hydro }}$ and $\mathbf{M}_{\text {hydro }}$, it is necessary to calculate the time derivative of the velocity potential $\varphi_{t}$, also called the acceleration potential. The direct way to compute $\varphi_{t}$ is by a backward finite-difference in time, which however has been proven to cause unexpected spurious oscillations in the time series of forces when the IBM is applied to a moving body $[49,56,47]$. Note that the spurious oscillation is not due to the known instability (see e.g. [58, 59]) related to the body motion equations, as it occurs even for forced oscillation problems. To avoid the instability associated with the equations of body motion, the Eulerian acceleration potential method (EAPM) has been proposed and also applied by many researchers $[60,58,61,59,62]$. We denote it as EAPM since an Eulerian description is implied in this approach. This approach has been proven to be very useful in improving accuracy and stability in prediction of large-amplitude motions of floating structures [63]. Since $\varphi_{t}$ also satisfies the Laplace equation, i.e. $\nabla^{2} \varphi_{t}=0$, it can be computed by solving another boundary value problem (BVP) in the same way as that for the velocity potential. The corresponding body-boundary condition is given as:

$$
\frac{\partial \varphi_{t}}{\partial n}=\left(\dot{\mathbf{U}}_{c}+\dot{\boldsymbol{\omega}}_{c} \times \mathbf{r}\right) \cdot \mathbf{n}+\left(\boldsymbol{\omega}_{c} \times \mathbf{n}\right) \cdot\left(\mathbf{U}_{c}-\nabla \varphi\right)-\left(\mathbf{U}_{c}+\boldsymbol{\omega}_{c} \times \mathbf{r}\right) \cdot(\mathbf{n} \cdot \nabla) \nabla \varphi,
$$

where $\dot{\mathbf{U}}_{c}$ is the translational acceleration of the moving body and $\dot{\boldsymbol{\omega}}_{c}$ is the angular acceleration about the rotation center. More details on the derivation of this equation can be found in [59, 62].

The last term in Eq. (20), associated with the second derivatives of the velocity potential, can be difficult to compute accurately. As it will be shown later, when the IBM is used, EAPM still introduces spurious oscillations, and it is not better than a simple backward finite-difference.

Alternatively, a method solving a BVP for a Lagrangian acceleration potential, proposed and applied by [64] and [65], seems to work very well. We will hereafter call it the Lagrangian acceleration 
so that we can define a BVP for $\Psi$ in the same way as the velocity potential, referred to in Eqs. (12) (14). On the free surface, the Dirichlet boundary condition is given as:

$$
\Psi=\mathbf{V} \cdot \nabla \varphi-\frac{1}{2}|\nabla \varphi|^{2}-g \eta
$$

At a moving body surface, the impermeable condition reads $\partial \varphi / \partial n=\mathbf{V} \cdot \mathbf{n}$. Meanwhile, according to [65], the following relation holds for $\Psi$

$$
\frac{\partial \Psi}{\partial n}=\frac{\partial}{\partial n}\left(\frac{\delta \varphi}{\delta t}\right)=\frac{\delta}{\delta t}\left(\frac{\partial \varphi}{\partial n}\right)=\frac{\delta}{\delta t}(\mathbf{V} \cdot \mathbf{n}) .
$$

Using the operator $\delta / \delta t=\partial / \partial t+\mathbf{V} \cdot \nabla$ yields

$$
\frac{\partial \Psi}{\partial n}=\dot{\mathbf{V}} \cdot \mathbf{n}+\mathbf{V} \cdot\left(\boldsymbol{\omega}_{c} \times \mathbf{n}\right)
$$

By direct comparison, this boundary condition for the Lagrangian acceleration potential $\Psi$ is simpler than that for the Eulerian acceleration potential in Eq. (20). In contrast to Eq. (20), Eq. (25) does not involve any derivatives of the velocity potential. This can significantly improve the accuracy of the calculation when using the IBM for a moving body. After the BVP for $\Psi$ is solved, we can obtain the the time derivative of the velocity potential by $\varphi_{t}=\Psi-\mathbf{V} \cdot \nabla \varphi$. It should be noted that this method can be applied not only to a rigid body but also to a flexible body. More details can be found in [66].

\section{Adaptive Cartesian grid system}

It has been shown, by Ma et al. [45], that the use of distorted cells in the 2D HPC method can compromise the global accuracy of the solver. As a general rule, square cells ensure the highest accuracy. In order to accurately model the rapid flow change near body boundaries or the free surface solely using square cells, local grid refinement is adopted in our study. Each original quadrant in these regions is successively subdivided into four equal-sized children, like quad-trees, until the size of refined cells reaches the predefined levels.

\subsection{Grid refinement procedure}

In this section, we will mainly use a Neumann boundary as an example to illustrate the procedure of grid refinement. The rigid boundary is firstly discretized and represented by a set of boundary points, and quad-tree cell refinement is carried out point by point sequentially. Initially, each body point belongs to a quadrant (or a cell) of the original grid system, and we assume that this point is 


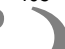

\section{Quadrant neighbors and node types}

In the quad-tree grid system, interpolation between different grid levels at the border nodes of each level is inevitably needed. Therefore, how to identify and interpolate border nodes becomes important. Normally, a quadrant has at least two and at most four neighbors. In this case, we can clearly identify border quadrants and interior ones: quadrants with four neighbors are interior while quadrants with two or three neighbors are on the border of this level.

Ma et al. [45] have proven that the accuracy of interpolation improves when the interpolating point is closer to the cell center. Therefore, from an accuracy point of view, the solution in border nodes should be interpolated from the central area of a cell. Commonly, this cell comes from the parent grid system. Fig. 6 sketches a typical quad-tree grid structure of two successive levels. The purple and yellow square nodes are typical fluid nodes from the parent and child grid system, respectively, and they naturally locate at the center of a cell on their own level.

Two types of border nodes between a parent and a child level are also shown in Fig. 6, while 


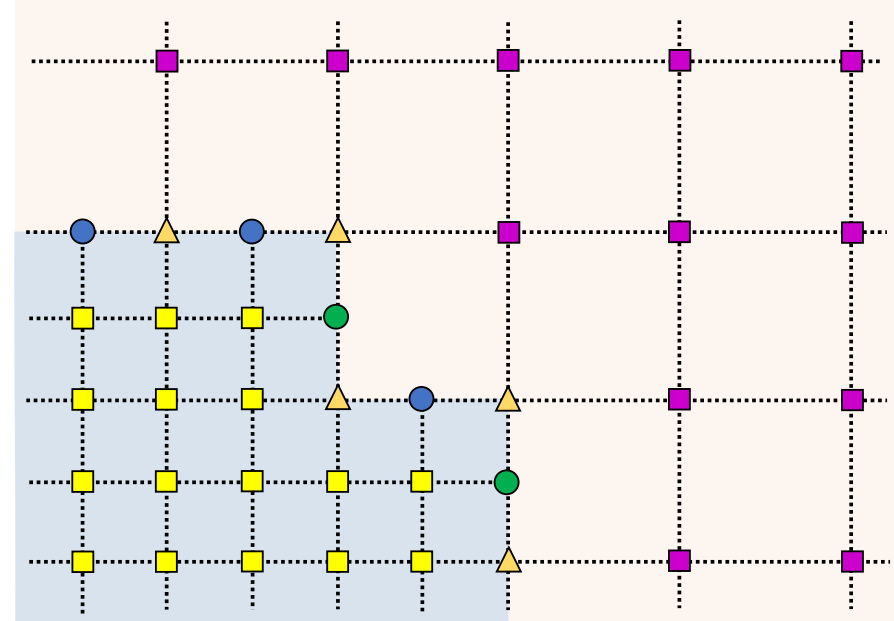

Fluid nodes on level-n

Fluid nodes on level- $(n+1)$

$\triangle$ Type-1 border nodes on level- $(n+1)$

Type-2a border nodes on level- $(n+1)$

Type-2b border nodes on level- $(n+1)$

Figure 6: Sketch of a typical quad-tree grid structure with different node types.

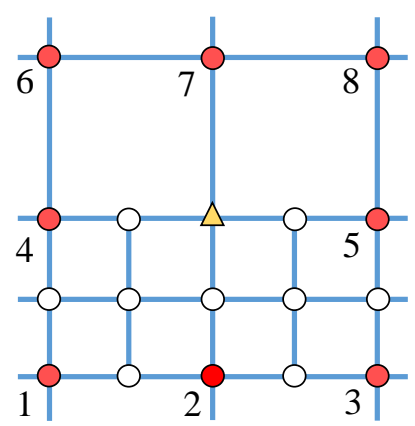

(a) Type-1 border node

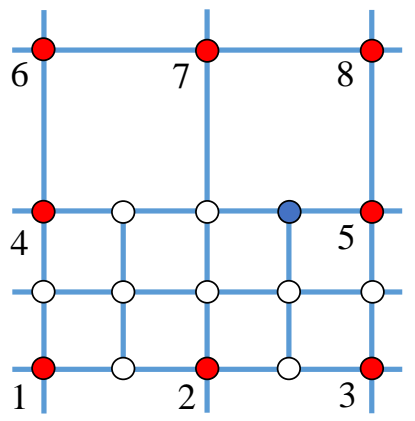

(b) Type-2a border node

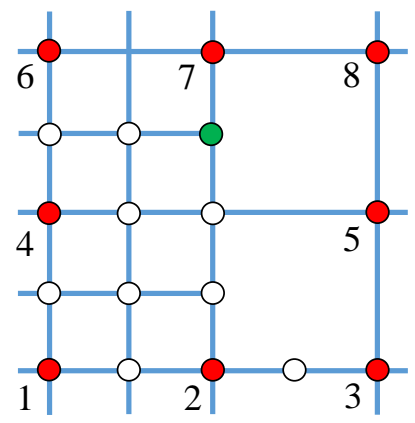

(c) Type-2b border node

Figure 7: Different types of border nodes using different interpolation strategies.

nodes, i.e. the brown triangles, coincide with nodes in the parent cell. Generally, they lie at the center of a parent cell. The red circular nodes are used for the interpolation while the white circles do not take part in the interpolating procedure. Type $2 \mathrm{a}$ or $2 \mathrm{~b}$ nodes do not coincide with nodes in the parent cell. Note that the red circular interpolating nodes in Fig. 7 come from the same parent cell, but in Fig. 7(a) and (b) index 1 5 are located on the child level while index $6 \sim 8$ are on the parent level, and in Fig. 7(c) index 1, 2, 4, 6, 7 are located on the child level while index 3, 5, 8 are on the parent level. Fig. 8 shows an overall distribution of node types on different levels, where the node types on the original grids are not displayed.

The global algebraic equation systems for both the velocity potential $\varphi$ and the Lagrangian or Eulerian acceleration potential share the same coefficient matrix $\mathcal{A}$. Fig. 9 shows an example of the coefficient matrix for a fixed circular cylinder in an infinite fluid shown in Fig. 8. The majority of the non-zero entries in $\mathcal{A}$ come from the connectivity equations Eq. (13), and are distributed close to the matrix diagonal. In the figure those entries marked along with a single integer (either 0,1 or 2) represent values from level-0, level-1 or level-2. Due to the presence of border nodes (Type- 1 and Type-2 in Fig. 7) between different levels (marked as $1 \rightarrow 0$ and $2 \rightarrow 1$ in Fig. 9) and some nodes of 


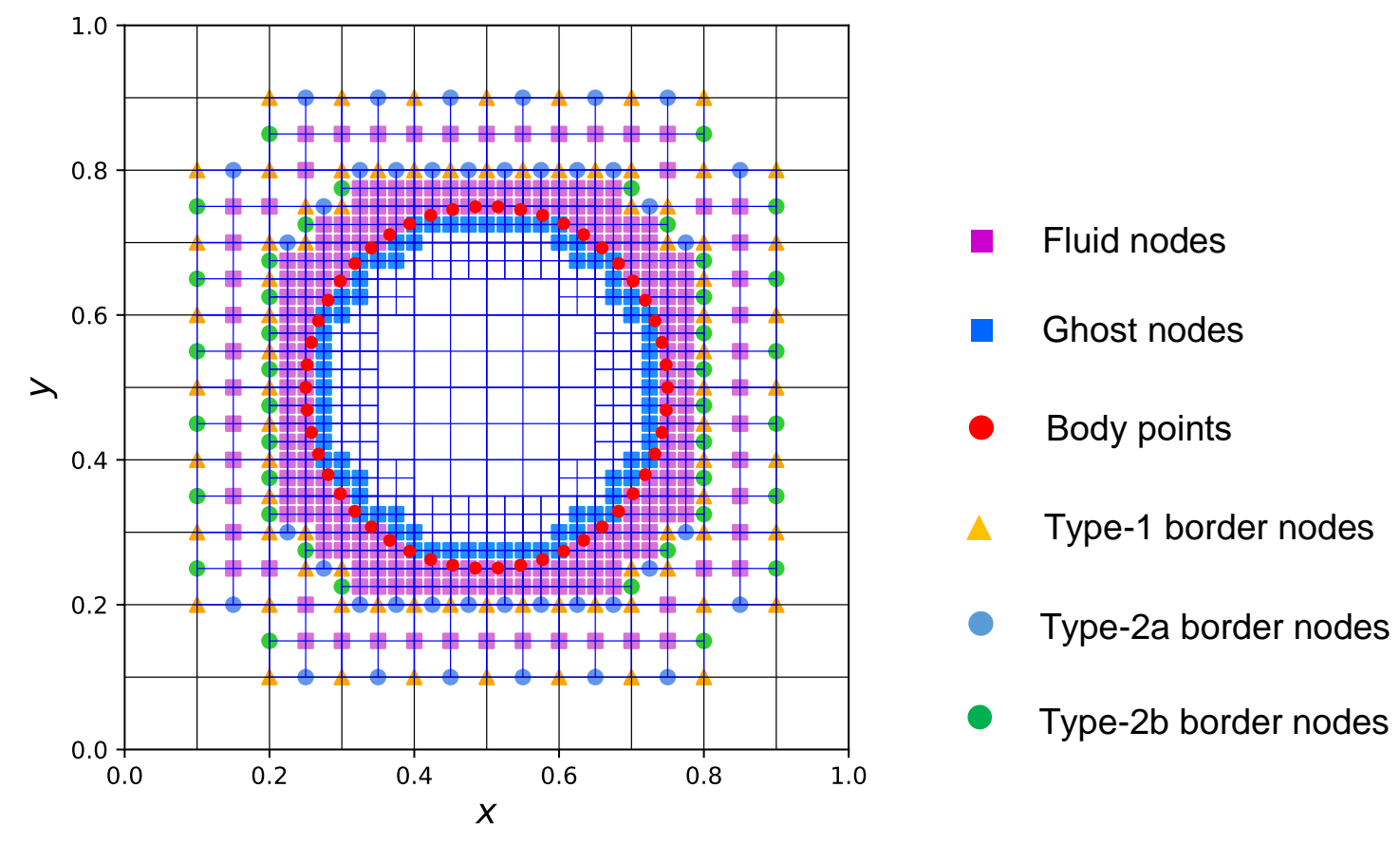

Figure 8: An overall view of an immersed circular cylinder and the associated adaptive cells with different levels and node types. The domain is $1 \times 1$ and the radius of the ring is 0.25 . The e-degree (1) $=1$ and the level $_{\max }=2$.

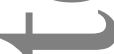

the parent cell but coming from deeper levels when building the connectivity equations (marked as 434 $0 \rightarrow 1$ and $1 \rightarrow 2$ in Fig. 9), there are also non-zero entries away from the diagonal of the matrix.

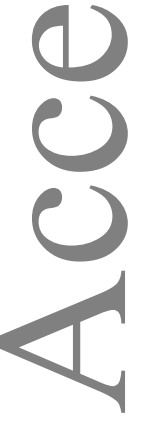




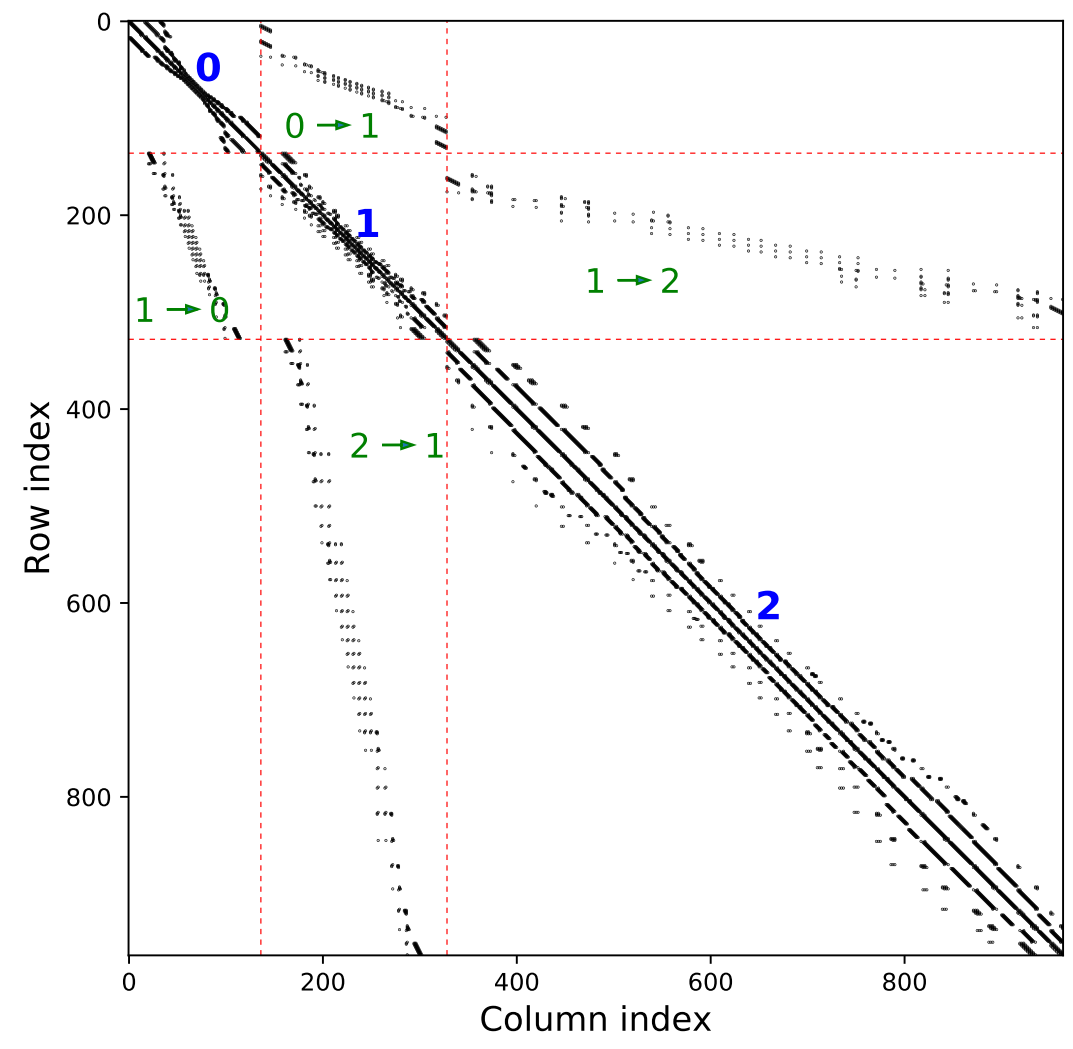

Figure 9: Example of global coefficient matrix in the IB-AHPC method with a rigid circular cylinder in an infinite fluid. Here, the $e$-degree $=2$ and the leve $_{\max }=2$. Level-0, level-1 and level-2 grid are for brevity denoted as 0,1 and 2 , respectively. $0 \rightarrow 1$ and $1 \rightarrow 0$ indicate coupling terms between the level- 0 and the level- 1 grid and vice versa, $1 \rightarrow 2$ and $2 \rightarrow 1$ indicate coupling terms between the level-1 and the level-2 grid.

\section{Accuracy and stability of the IB-AHPC method}

\subsection{Accuracy and spatial convergence analysis}

\section{5.1.1 A fixed circular cylinder in an infinite fluid with a uniform oscillatory flow}

Here, we consider a fixed submerged circular cylinder with radius $R$ in an infinite fluid domain with a uniform oscillatory flow $U(t)=U_{0} \cos (\omega t)$. Referring to Fig. 10, a rectangular computational domain of $l_{x} \times l_{y}$ is chosen, and the center of the cylinder is coincident with the center of the domain. ${ }_{41}$ The origin of an Earth-fixed coordinate system, Oxy, is located at the southwestern corner of the 443 is given as [67]:

$$
\varphi_{\text {ana }}(x, y, t)=U_{0} \cos (\omega t)\left(x-\frac{l_{x}}{2}\right)\left[1+\frac{R^{2}}{\left(x-\frac{l_{x}}{2}\right)^{2}+\left(y-\frac{l_{y}}{2}\right)^{2}}\right] .
$$

444 This analytical solution will be used as boundary conditions along Dirichlet boundaries in Fig. 10 .

${ }_{445}$ The Neumann boundary condition $\partial \varphi / \partial n=\mathbf{U}_{b o d y} \cdot \mathbf{n}=0$ is applied on the rigid cylinder. The 


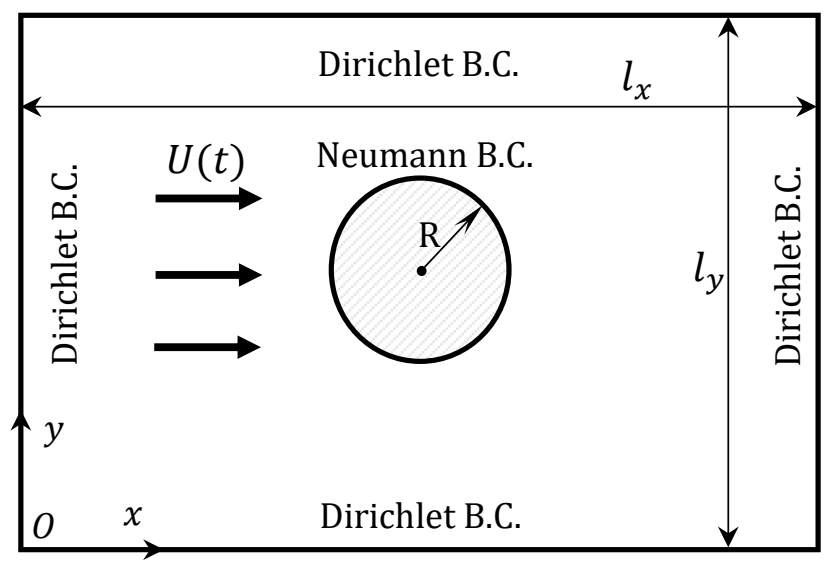

Figure 10: An illustration of a fixed circular cylinder in the computational fluid domain with a uniform oscillatory flow.

analytical solution of the inline force on the cylinder can be expressed as

$$
F_{a n a}=-\mu_{a n a} \frac{d U}{d t} .
$$

Here $\mu_{\text {ana }}$ is an inertia coefficient

$$
\mu_{\text {ana }}=2 \pi \rho R^{2},
$$

where $\rho$ is the fluid density. The $L_{2}$-error is used as a measure of the accuracy of our numerical calculations, which for a general variable $G$ is expressed as:

$$
L_{2, G}=\sqrt{\frac{\sum_{i=1}^{N}\left(G_{n u m, i}-G_{\text {ana }, i}\right)^{2}}{\sum_{i=1}^{N} G_{a n a, i}^{2}}} .
$$

In this case, the grid size in the deepest level, $d x=\Delta x(1 / 2)^{\text {level }_{\max }}$, is applied, where the original grid size, $\Delta x / R=0.42$, is chosen. In order to match the maximum level, the size of the boundary elements are chosen as the same as the finest grid size, i.e., $\Delta s \approx \sqrt{2} \Delta x(1 / 2)^{\text {level }_{\max }}$.

In the following analyses, the dimension of the fluid domain is chosen as $l_{x}=l_{y}=6 R$. Fig. 11 shows the $L_{2}$ norm of the error for both the velocity potential on the body points and the inertia constant $\mu$ with different e-degree. The four different grid size stands for four levels, i.e. level $=$ $1 \sim 4$, of the quad-tree system with the same original grid size. The convergence for the velocity potential on all body nodes, shown on the left-hand side of Fig. 11, is between $3^{\text {rd }}$ and $4^{\text {th }}$ order versus the non-dimensional grid size on the deepest level, i.e., $L_{2, \varphi} \sim(R / d x)^{-3.5}$. The convergence rate is similar to the original HPC method in [24,44], indicating that the present IB-AHPC with adaptive cells does not compromise the accuracy of the HPC method. Similarly, the error for the calculated inertia coefficient converges at a rate $L_{2, \mu} \sim(R / d x)^{-3.5}$, which agrees well with the accuracy for the velocity potential. We can, moreover, discover that the larger the e-degree is, the faster the convergence rate will be for the velocity potential, but the improvement is very marginal. The $e$ degree also shows negligible influence on the convergence rate for the inertia coefficient, as shown in the right sub-plot in Fig. 11.

Fig. 12 plots the total number of active nodes as a function of $R / d x$ for different levels of cell refinement and different e-degree. The total number of grid nodes for a grid system without local 

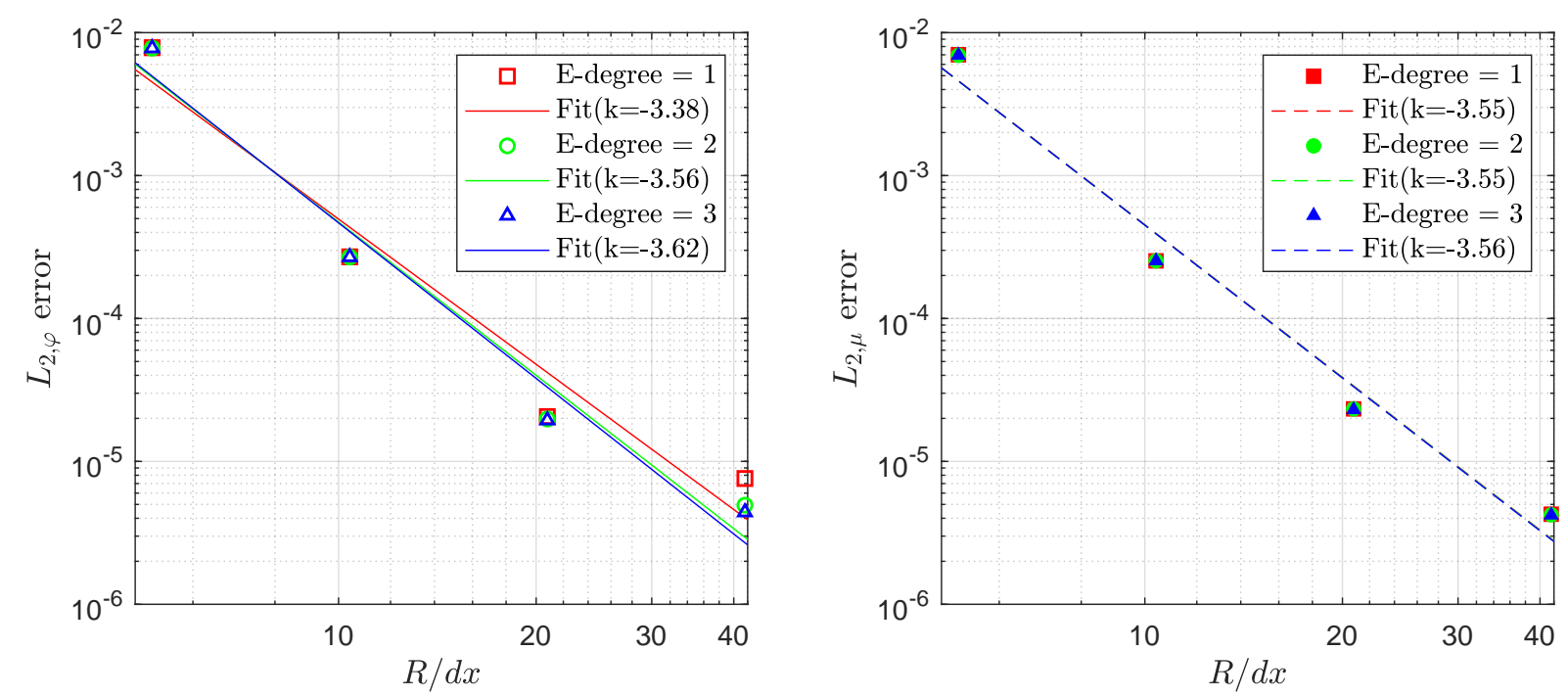

Figure 11: Grid convergence for the solution of a fixed submerged cylinder in a uniform oscillatory flow with different e-degree. Left: Velocity potential $\varphi$ on body. Right: Inertia coefficient $\mu$. Here, $d x$ represents the grid spacing in the deepest level grid.

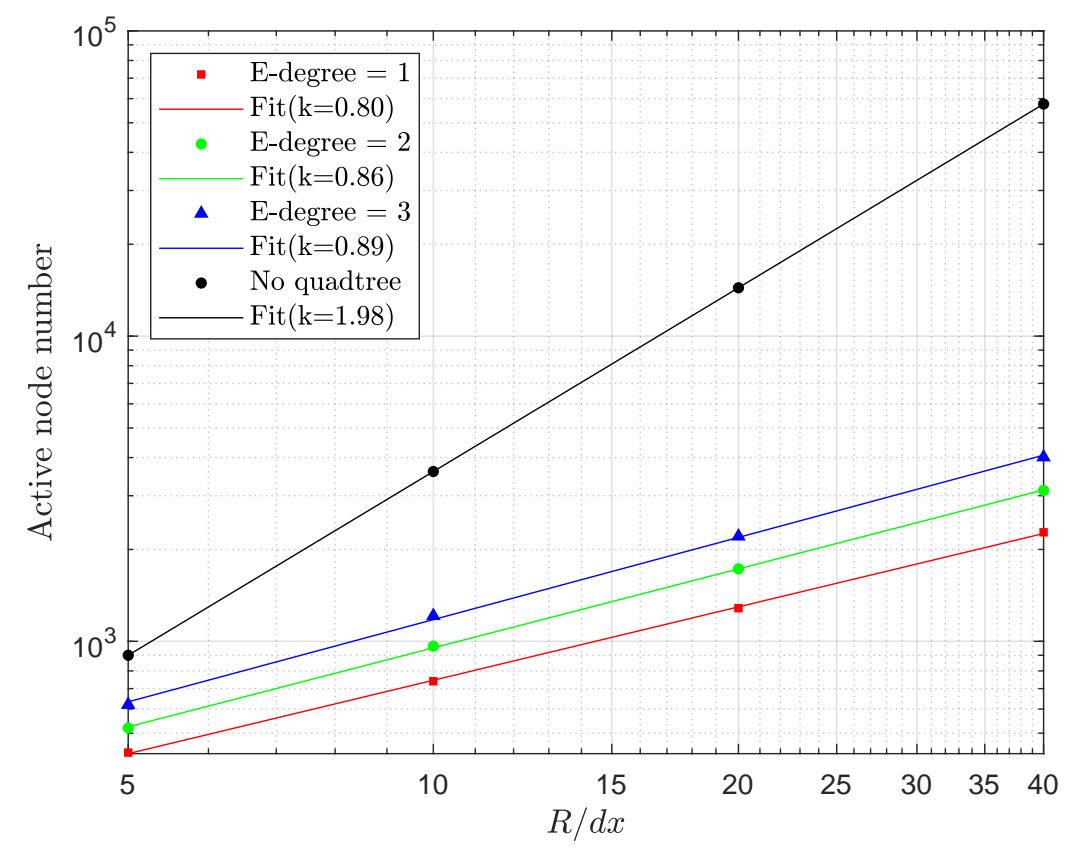

Figure 12: Number of active nodes (unknowns in the global matrix equations) versus grid size for adaptive cells with different $e$-degree and cells without quad-tree refinements.

refinement is also included in the figure for comparison. The fitted rate $k$ indicates that, with local cell refinement, the number of unknowns can be dramatically reduced, especially when the local grid is significantly refined or for large computational domains. For the considered example, the total number of grid points required to reach a given accuracy of solution is found to scale with $(1 / \Delta x)^{0.8 \sim 0.9}$ compared to $(1 / \Delta x)^{2}$ for a method without local grid refinement. Since the number of active nodes is directly associated with the computational cost in solving the global equations, it 


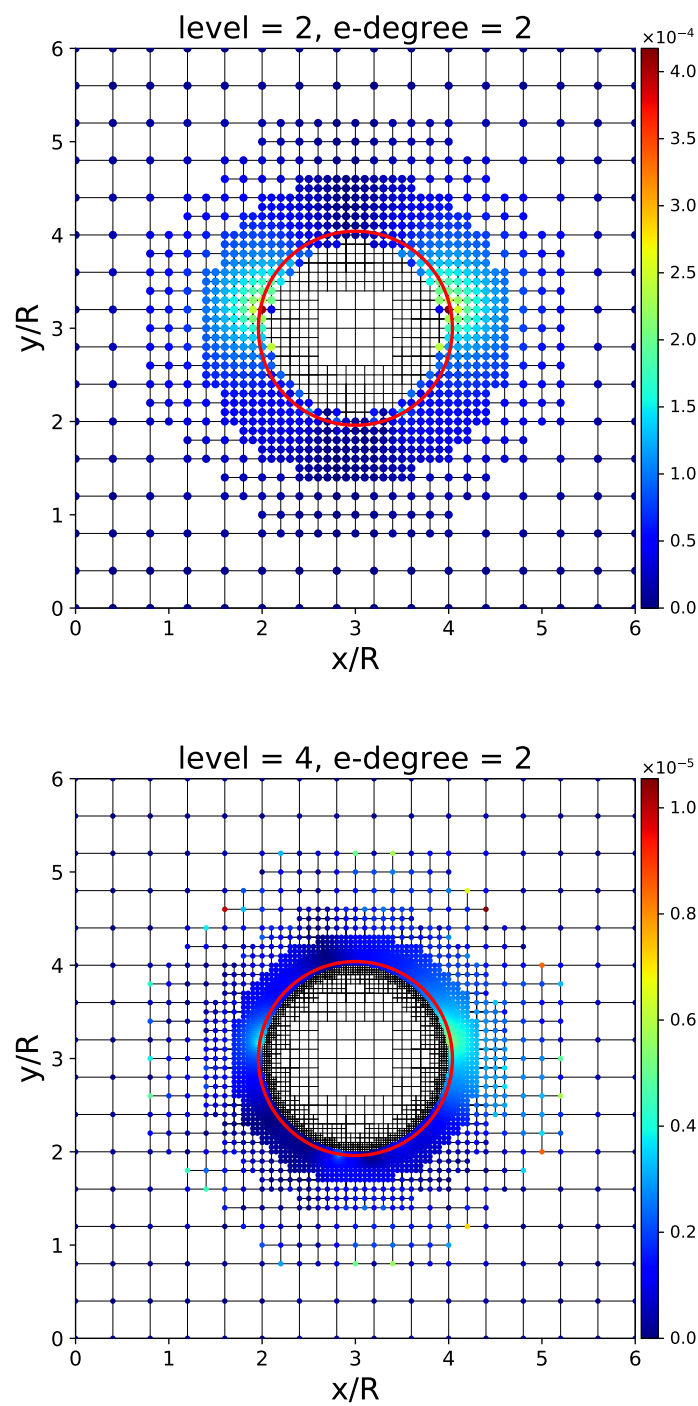

Figure 13: Distribution of $e_{\varphi}$ in vicinity of cylinder for different maximum level, e-degree $=2$.

Fig. 13 shows the relative error distribution for the velocity potential in the whole computational domain for different levels of quad-tree refinement. The relative error is defined as $e_{\varphi}=$ $\left|\varphi_{\text {ana }}-\varphi_{\text {num }}\right| / \max \left(\left|\varphi_{\text {ana }}\right|\right)$ for each active node, where $\max \left(\left|\varphi_{\text {ana }}\right|\right)$ is the maximum absolute analytical potential in the entire domain. Actually, the error tends to be largest near the body boundary, which can be seen from Fig. 13 for level $=1$ or 2 . But due to adopting the mode of interpolation for Type-2 border nodes in Fig. 7, if given a closer look at these border nodes between level-0 and level- 1 for the case of level $=4$, we can notice that the relative error of some Type- 2 nodes tends to be larger than that around the body boundary. But this phenomenon disappears between deeper levels, namely between level-3 and level-4, which might result from the large truncate error in coarse grid. On the other hand, the error of Type-1 nodes seems to be smaller than that of two adjacent Type-2 nodes. This can be explained, from a finite difference point of view, by the fact that the 
surge is given as: serge is given as:

accuracy of central difference schemes is better than that for off-centered schemes. In other words, the accuracy of interpolation for the HPC method is always the best at the center of a cell.

\subsubsection{An oscillating circular cylinder in an infinite fluid}

Hanssen et al. [49] in their previous study showed that applying the IBM for a moving structure could produce spurious pressure-force oscillations. This issue was solved by Hanssen [47] applying an overlapping-grid strategy and solving an extra BVP for the time derivative of the velocity potential, however, with a cost of increased complexity of numerical implementation and somewhat larger computational effort. In this section, we show that the spurious force oscillations can be eliminated by using the Lagrangian acceleration potential method (LAPM) described in $\S 3.3$ when applying the IBM to a moving structure.

To demonstrate this, we consider the forced harmonic oscillation of the circular cylinder in an infinite fluid, which has also been studied by Hanssen et al. [49]. The cylinder is initially set in the center of the computational domain with $l_{x}=l_{y}=20 R$. The motion of the center of the cylinder in

$$
\left\{\begin{array}{l}
x_{c}(t)=\frac{l_{x}}{2}+\frac{U_{c}}{\omega} \sin \omega t \\
y_{c}(t)=\frac{l_{y}}{2}
\end{array}\right.
$$

Here $U_{c}$ is velocity amplitude, $\omega$ is the oscillatory frequency. The motion amplitude is set to be $U_{c} / \omega=4 R$. The analytical solution for the velocity potential in this case is

$$
\varphi_{\text {ana }}(x, y, t)=-U_{c} \cos (\omega t) \cdot\left(x-x_{c}(t)\right)\left[\frac{R^{2}}{\left(x-x_{c}(t)\right)^{2}+\left(y-y_{c}(t)\right)^{2}}\right] .
$$

The analytical linear hydrodynamic force on body surface due to the harmonic oscillation is

$$
f_{x}(t)=-\rho \oint_{\Gamma_{S}} \frac{\partial \varphi}{\partial t} n_{x} d s=f_{0} \sin (\omega t)
$$

where $f_{0}=\rho \pi \omega R^{2} U_{c}, \rho$ is the fluid density and $n_{x}$ is the horizontal component of the normal vector on the surface $\mathbf{n}=\left(n_{x}, n_{y}\right)$, defined as positive pointing into the body.

The grid spacing in this case is the same as that for the fixed cylinder case. level $l_{\text {max }}$ ranging from 1 to 4 is applied to study the convergence of $f_{x}$. The time step $\Delta t=T / 252=0.05 \mathrm{~s}$, and three different velocity amplitudes are considered: $U_{c}=1.0 \mathrm{~m} / \mathrm{s}, 2.0 \mathrm{~m} / \mathrm{s}$ and $2.5 \mathrm{~m} / \mathrm{s}$. Here $T=2 \pi / \omega$. Analytical Dirichlet boundary conditions are applied along the four boundaries of the computational domain. Fig. 14 shows the $L_{2}$ error of $f_{x}$ with three different velocity amplitudes as well as the error of the inertia coefficient for a fixed cylinder for comparison. It is expected that the oscillating velocity amplitude has almost no impact on the convergence rate. Although the error for fixed cases is smaller than that for oscillating cases, the convergence rates of $f_{x}, L_{2, f_{x}} \sim(R / d x)^{-3.0 \sim-4.0}$, for the oscillation cases are in the same range as that for a fixed cylinder, which is different from the observation in [49].

Hanssen et al. [49] and Kontos [56] have computed the acceleration potential $\varphi_{t}$ using the finite difference method (FDM), and obtained highly oscillatory force signals. These spurious oscillations can be reduced by increasing the time step size and by decreasing grid spacing. Here, we also compute the acceleration potential by applying the EAPM and the LAPM, as described in $\S 3.3$. In both cases, the moving velocity amplitude $U_{c}=1.0 \mathrm{~m} / \mathrm{s}$. We also set two time steps $\Delta t=T / 1260=0.01$ $\mathrm{s}$ and $\Delta t=T / 252=0.05 \mathrm{~s}$. As shown in Fig. 15 (left column) for the LAPM, the time series of force on the harmonically oscillating cylinder agree very well with the analytical solution from 


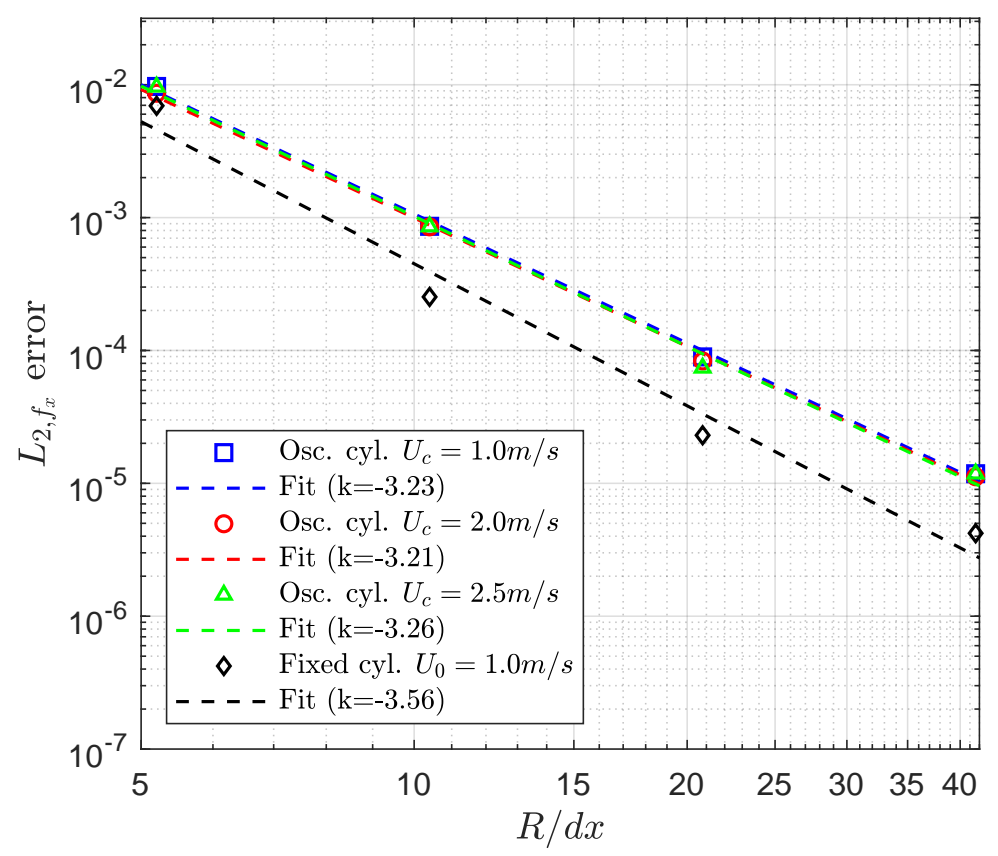

Figure 14: Grid convergence of $f_{x}$ on an oscillating submerged cylinder with three different amplitudes of velocity in undisturbed fluid. The error of the inertia coefficient $L_{2, \mu}$ for a fixed cylinder is also demonstrated for comparison. Here, $d x$ represents the grid spacing in the deepest level grid.

Eq. (33). We also include in this plot the results obtained by using the EAPM (middle column) and a simple first-order backward FDM (right column). The results demonstrate that the LAPM is a very promising approach in eliminating the force oscillations, and its robustness is independent of the time step size, in contrast to the EAPM and the FDM. Improving the local grid refinement from level $_{\max }=1$ to 3 is also seen to reduce the oscillations for both the EAPM and the FDM results, especially for larger time steps, but somehow we can tell that the $1^{\text {st }}$-order FDM seems to perform slightly better than the EAPM in this case.

Fig. 16 compares the CPU time per time step of generating global matrix system and solving global equations for $\varphi$ and $\varphi_{t}$ by using the FDM, the EAPM and the LAPM. Note that, for brevity, $\varphi_{t}$ here, represents either the Lagrangian or the Eulerian acceleration potential. In the plot, we also displayed the CPU time per time step by using different grid size, i.e. different grid level $_{\max }$. In practice, the BVPs for $\varphi$ and $\varphi_{t}$ share the same global coefficient matrix, then extra time needs to be spent on the generation of the right-hand-side vector for $\varphi_{t}$, and on solving the equations. We can find that the time spent for the EAPM and the LAPM is almost the same even if it is slightly higher for the EAPM, and the time is nearly twice of that for the FDM.

\section{$5.2 \quad$ Stability analysis}

To assess the stability of the present IB-AHPC method, a matrix-based stability study is conducted in this section. Here, we consider a linear numerical wave tank without any structures in the fluid or at the free surface. We consider the linearized kinematic and dynamic free-surface conditions in matrix form in $2 \mathrm{D}$ :

$$
\frac{\partial}{\partial t}\left[\begin{array}{l}
\eta \\
\tilde{\varphi}
\end{array}\right]=\left[\begin{array}{cc}
0 & \frac{\partial}{\partial z} \\
-g & 0
\end{array}\right]\left[\begin{array}{l}
\eta \\
\tilde{\varphi}
\end{array}\right]
$$



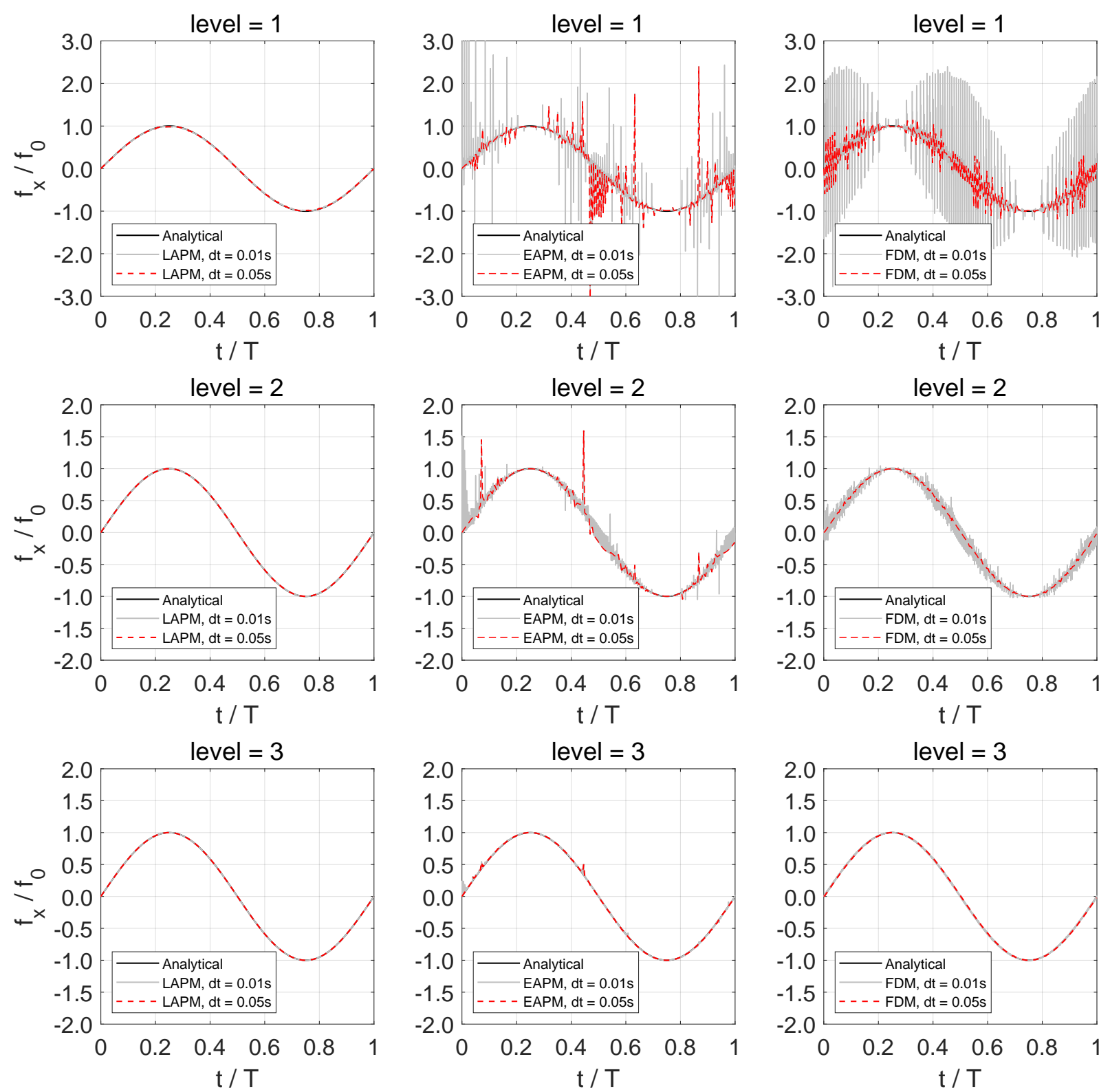

Figure 15: Time series of force $f_{x}$ on an oscillating circular cylinder in an infinite still fluid using the LAPM (left), the EAPM (middle) and the FDM (right) for different level ${ }_{\text {max }}$, respectively.

where $\eta$ is the surface elevation and $\tilde{\varphi}=\varphi(x, 0, t)$ is the velocity potential at the free surface. The free surface will be discretized into a set of wave markers, as described in $\S 3.2$. As a result, a space-discretized form of Eq. (34) can be rewritten as

$$
\frac{\partial}{\partial t}\left[\begin{array}{l}
\boldsymbol{\eta} \\
\tilde{\boldsymbol{\varphi}}
\end{array}\right]=\left[\begin{array}{cc}
0 & \mathcal{L} \\
-g \mathcal{I} & 0
\end{array}\right]\left[\begin{array}{l}
\boldsymbol{\eta} \\
\tilde{\boldsymbol{\varphi}}
\end{array}\right] \Rightarrow \frac{\partial \mathbf{f}}{\partial t}=\mathcal{J} \mathbf{f} .
$$

${ }_{547}$ Here the vector $\mathbf{f}=\left[\eta_{1}, \eta_{2}, \ldots, \eta_{N_{m}}, \tilde{\varphi}_{1}, \tilde{\varphi}_{2}, \ldots, \tilde{\varphi}_{N_{m}}\right]^{T}$ is the combination of the free-surface elevation

548

549

550

551 and velocity potential at all wave markers. The identity matrix $\mathcal{I}$ and the operator matrix $\mathcal{L}$ are of size $N_{m} \times N_{m}$, where $N_{m}$ is the number of wave markers. $\mathcal{J}$ denotes the Jacobian matrix. In the global linear equations system $\mathcal{A} \varphi=\mathrm{e}$ with $N$ unknowns, $\tilde{\varphi}$ can be distributed in the corresponding positions in the right-hand side vector $\mathbf{e}$ in the form of 


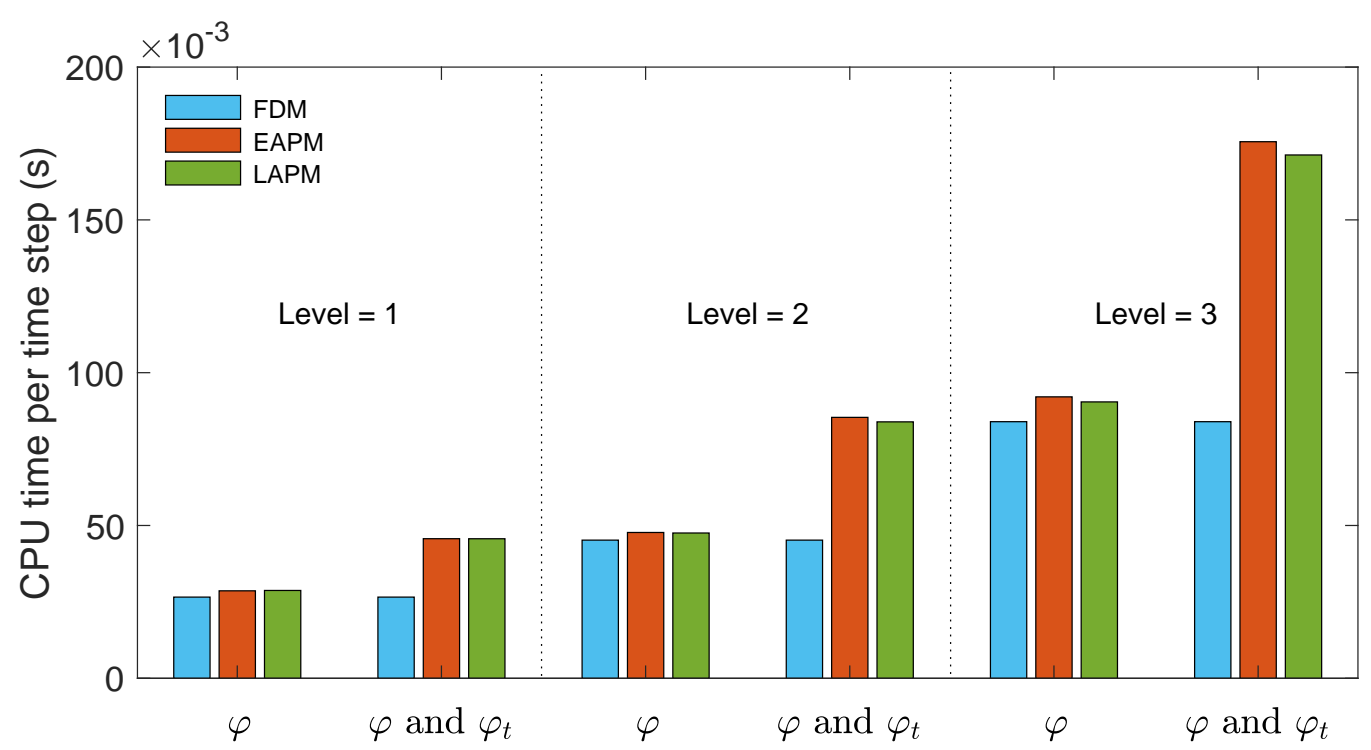

Figure 16: CPU time per time step for generating the global matrix system and solving the quations for $\varphi$, and total CPU time per time step for generating the global matrix system and solving equations for $\varphi$ and $\varphi_{t}$ by using the FDM, the EAPM and the LAPM with different level $l_{\text {max }}$. Here, $\varphi_{t}$ represents either the Eulerian or the Lagrangian acceleration potential.

$$
\mathcal{Q}_{N \times N_{m}} \tilde{\boldsymbol{\varphi}}_{N_{m} \times 1} \simeq \mathbf{e}_{N \times 1}
$$

by an operator matrix $\mathcal{Q}$. Note that the left-hand side and the right-hand side of Eq. (36) are not exactly the same, with a constant term missing, which however is independent of $\tilde{\varphi}_{N_{m} \times 1}$. Neglecting this constant term will not influence the results of the stability analysis. Hence, we can also get the following relations:

$$
\mathcal{A}_{N \times N}^{-1} \mathcal{Q}_{N \times N_{m}} \tilde{\boldsymbol{\varphi}}_{N_{m} \times 1} \simeq \boldsymbol{\varphi}_{N \times 1} .
$$

After taking the vertical derivatives of the velocity potential $\varphi_{N \times 1}$ by an operator matrix $\mathcal{D}$ :

$$
\left(\mathcal{D}_{N_{m} \times N} \mathcal{A}_{N \times N}^{-1} \mathcal{Q}_{N \times N_{m}}\right) \tilde{\boldsymbol{\varphi}}_{N_{m} \times 1} \simeq \mathcal{D}_{N_{m} \times N} \boldsymbol{\varphi}_{N \times 1}
$$

we finally obtain the operator matrix $\mathcal{L}$ in the form of

$$
\mathcal{L}=\mathcal{D} \mathcal{A}^{-1} \mathcal{Q}
$$

For a given discrete system, we can compute the operator matrix $\mathcal{L}$ and hence complete the Jacobian matrix $\mathcal{J}$ in Eq. (35). For the discretization of the free-surface boundary conditions discussed above, the eigenvalues of the matrix $\mathcal{J}$ are purely imaginary and can be calculated numerically. To guarantee stability, the maximum eigenvalue of matrix $\mathcal{J}$ multiplied by the time step $\Delta t$ must be inside the stability region for a given time stepping scheme. For the $4^{\text {th }}$-order explicit Runge-Kutta (RK4) scheme used in our study, the stability contour can be expressed as

$$
\beta(\lambda \Delta t)=1+\lambda \Delta t+\frac{(\lambda \Delta t)^{2}}{2 !}+\frac{(\lambda \Delta t)^{3}}{3 !}+\frac{(\lambda \Delta t)^{4}}{4 !},
$$

${ }_{564}$ where $\lambda$ is an eigenvalue of matrix $\mathcal{J}$. For a stable solution, we need to have 


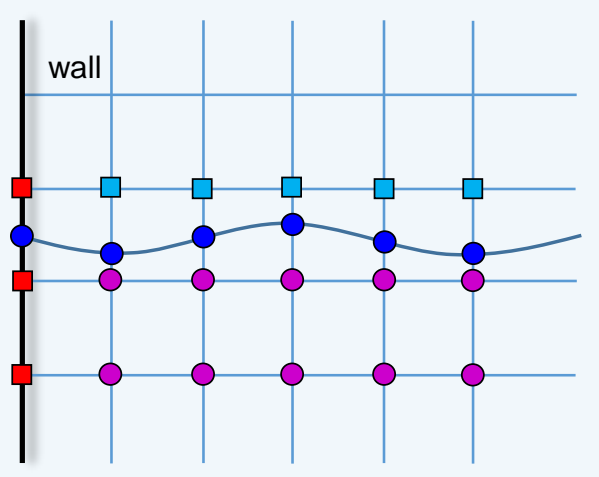

Strategy-1

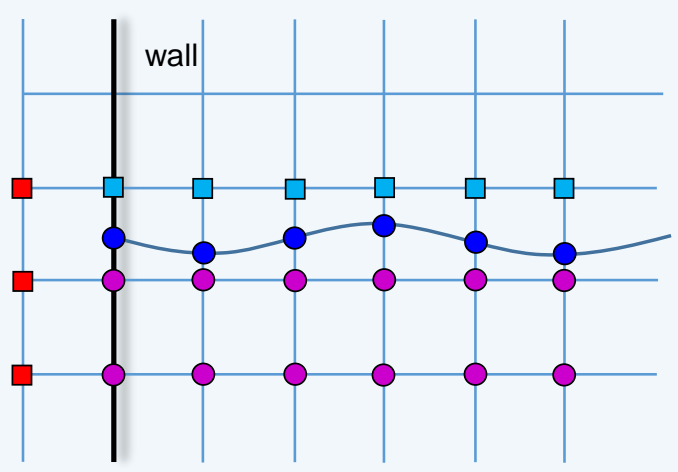

Strategy-2

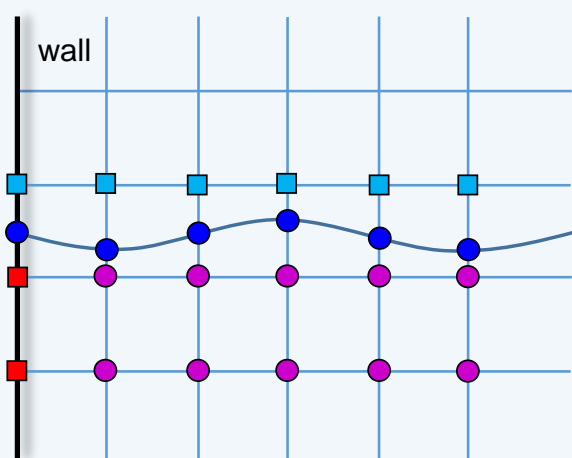

Strategy-3 $\square \quad$ Ghost nodes - FS B.C.

- Wave markers

Wall points - Neumann B.C.

- Fluid nodes

Figure 17: Three strategies to deal with markers on walls.

$$
|\beta(\lambda \Delta t)| \leq 1
$$

Since the eigenvalues of $\mathcal{J}$ are purely imaginary, the contour of Eq. (40) intersects with the imaginary axis at $\pm 2 \sqrt{2} \mathrm{j}$, where $\mathrm{j}=\sqrt{-1}$, i.e. $\left|\lambda_{\max }\right| \Delta t \leq 2 \sqrt{2}$.

Up to this point, the influence of grid spacing, time stepping and choice of boundary conditions on the stability can be explored by this stability analysis procedure. In general, the instability problem always tends to happen at the free surface, particularly at the intersection between the wave profile and a Neumann boundary. To deal with the wave marker along the left wall boundary, Strategy-3 in Fig. 17 was first adopted where the ghost node above this wave marker is regarded as a Dirichlet boundary node using the free surface boundary conditions. However, instability occurs when this strategy is applied, even for generating waves with very small wave slope and amplitude. This kind of instability always starts with markers on the left and right walls oscillating irregularly and eventually leads to the breakdown of the computation. In order to increase the stability of the whole system, two other strategies are put forward, i.e. Strategy-1 and Strategy-2 in Fig. 17. For Strategy-1, the ghost node above the wave marker on the wall will be regarded as a Neumann boundary node, but shares the coordinates of this wave marker when producing the global linear equation system. This kind of strategy is very easy to implement. In Strategy-2, the ghost node with respect to the wave marker on the wall is treated as in Strategy-3, while the original wall nodes are regarded as fluid nodes and a set of ghost nodes is added outside of the wall sharing information from the corresponding nodes on the wall. The difference between Strategy-2 and Strategy-3 is that the wave marker in Strategy-2 
Table 2: Maximum eigenvalues $\lambda_{\max }$ of three strategies for different maximum level of quadtree and e-degree.

\begin{tabular}{lccccc}
\hline & e-degree & \multicolumn{4}{c}{ level $_{\text {max }}$} \\
\cline { 3 - 5 } & 1 & 1 & 2 & 3 & 4 \\
\hline Strategy-1 & 2 & $0 \pm 11.66 \mathrm{j}$ & $0 \pm 16.49 \mathrm{j}$ & $0 \pm 23.32 \mathrm{j}$ & $0 \pm 32.98 \mathrm{j}$ \\
& 3 & & & \\
\hline & 1 & & & \\
Strategy-2 & 2 & $0 \pm 11.66 \mathrm{j}$ & $0 \pm 16.49 \mathrm{j}$ & $0 \pm 23.32 \mathrm{j}$ & $0 \pm 32.98 \mathrm{j}$ \\
& 3 & & & & \\
\hline & 1 & $0 \pm 11.66 \mathrm{j}$ & $0 \pm 16.49 \mathrm{j}$ & $0 \pm 23.32 \mathrm{j}$ & $0 \pm 1.65 \times 10^{9} \mathrm{j}$ \\
Strategy-3 & 2 & $0 \pm 2.20 \times 10^{8} \mathrm{j}$ & $0 \pm 2.25 \times 10^{8} \mathrm{j}$ & $0 \pm 2.37 \times 10^{8} \mathrm{j}$ & $0 \pm 2.42 \times 10^{8} \mathrm{j}$ \\
& 3 & $0 \pm 1.95 \times 10^{8} \mathrm{j}$ & $0 \pm 2.24 \times 10^{8} \mathrm{j}$ & $0 \pm 2.14 \times 10^{8} \mathrm{j}$ & $0 \pm 2.91 \times 10^{8} \mathrm{j}$ \\
\hline
\end{tabular}

Table 2 gives the maximum eigenvalues from the matrix-based stability analysis for these three strategies of handling wave markers on walls with different $e$-degree and depth of grid level. From this table, an unexpected observation is that Strategy-1 and Strategy-2 share the same eigenvalues with the same grid refinements; here, it is very clear and reasonable that the e-degree plays a minor role in influencing the stability. On the other hand, we can see that most of the eigenvalues for Strategy3 are many orders of magnitude larger than that for the other strategies. This stability analysis of Strategy-3 provides a mathematical perspective to explain the instability occurring during the computation. Although the eigenvalues for Strategy-3 at e-degree $=1$, level $_{\max }=1 \sim 3$ are the same as Strategy-1 and Strategy-2, we have to mention that the calculation of the linear waves fails to match perfectly with the analytical solution while the other two strategies give good results.

Since Strategy-1 and Strategy-2 share the same maximum eigenvalues, we can assume that they will exhibit the same stability behavior. Fig. 18 shows the eigenvalues of the Strategy-1 distributed within the stable region of the RK4 scheme for different level $_{\max }$ of the quad-tree. All eigenvalues in this linear stability analysis are on the imaginary axis, and the horizontal distance between the numerical stability eigenvalue and the stability contour of the RK4 scheme indicates how much numerical diffusion is introduced. In this case, although this strategy is stable for all grid refinements, it can be predicted that the numerical diffusion introduced is the least with the finest grid and the most with the coarsest grid, since not all eigenvalues are on the RK4 contour when level $=1$.

As discussed by Engsig-Karup et al. [33], the continuous eigenvalue of the Jacobian matrix $\mathcal{J}$ in Eq. (35) is simply the wave circular frequency $\sqrt{g k \tanh (k h)}$, where $g$ is the gravitational acceleration, $k$ is the wave number and $h$ is mean water depth. Therefore, we plot the maximum eigenvalues normalized by the Nyquist circular frequency $\omega_{N}=\sqrt{g k_{N} \tanh \left(k_{N} h\right)}$, versus the normalized grid size $L_{w} / \Delta x$ ( $L_{w}$ is wave length) for Strategy-1 and -2 in Fig. (19)(a). Here the four red circles correspond to the four different level $_{\max }$ of the grid system. The Nyquist wave number is defined as $k_{N}=\pi / \Delta x$. Noticeable is that the normalized maximum eigenvalues are independent of the grid 

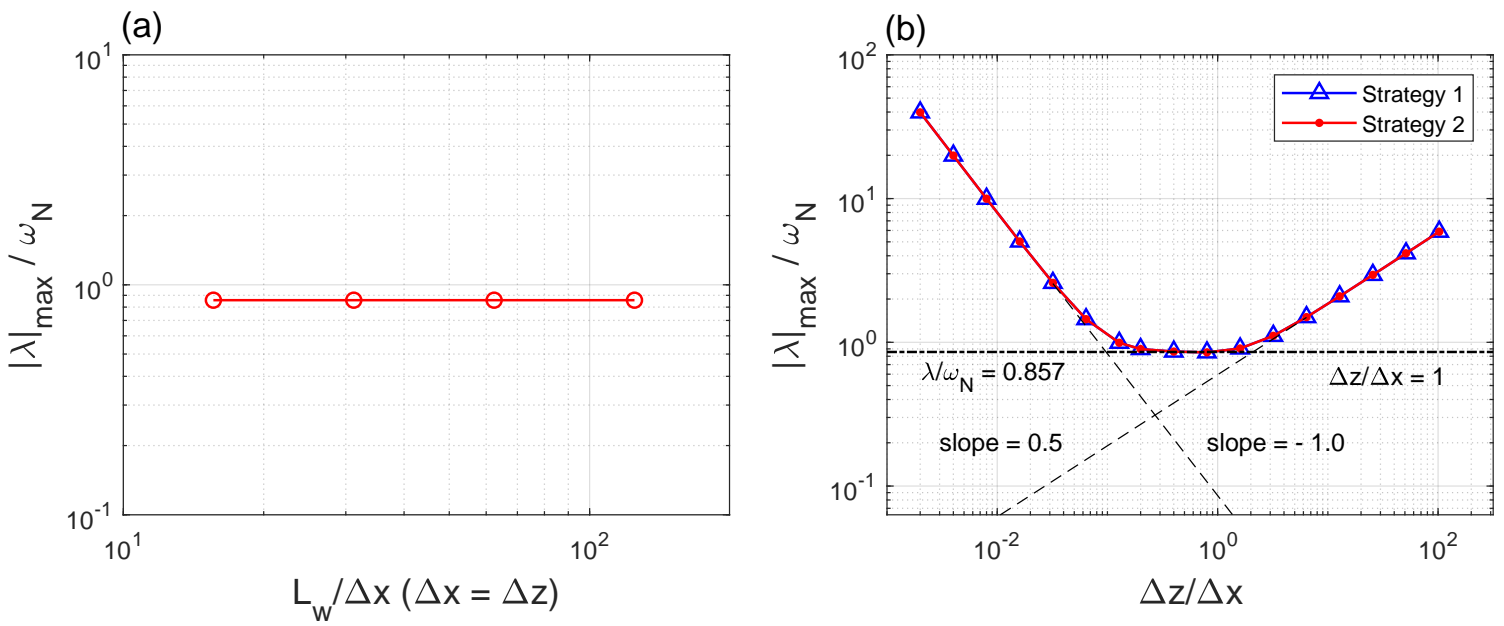

Figure 19: (a) Maximum eigenvalues vs. grid size; (b) Maximum eigenvalues vs. mesh aspect ratio for Strategy-1 and Strategy-2.

to distorted or stretched cells according to the study by Ma et al. [45]. These investigations and observations provide a strong motivation for using cells with mesh aspect ratio $\Delta z / \Delta x=1$. Another point is that Strategy-1 and Strategy-2 share the same stability performance. Considering the much easier implementation of Strategy-1, this strategy will be used in the following wave-body interaction study.

\section{(628) 6 Numerical applications}

\subsection{Generation of periodic waves in a nonlinear wave tank}

In this section, generation of periodic waves in a fully-nonlinear numerical wave tank with finite water depth is considered. Fig. 20 shows a sketch of the numerical wave tank, where we in the present study use a wave-making zone $L_{W M Z}=L_{w}$ and wave-damping zone $L_{W D Z}=2.3 L_{w}$, where $L_{w}=2.6$

${ }_{633} \mathrm{~m}$ is the wave length. $\partial \Omega_{\text {left }}, \partial \Omega_{\text {right }}$ and $\partial \Omega_{\text {seabed }}$ are Neumann boundaries. The length of this wave tank is $L=20 \mathrm{~m}$ and the water depth is $h=2.5 \mathrm{~m}$. A global Cartesian coordinate system $O x z$ is located on the mean water line at the middle of this tank with $z$-axis pointing upwards. The free surface is represented by a set of wave markers, which are only allowed to move vertically. Namely, the semi-Lagrangian free-surface tracking technique is applied. More details on the semi-Lagrangian method of dealing with the free-surface dynamic and kinematic free-surface conditions can be found in, e.g. [51] and [47]. The number of wave markers is equal to the number of grid points along the $x$-axis in the deepest level.

The velocity potential and elevation of the free surface from the high-order solution of stream function by [68] or [69] are given as input signals at each time step. One could also refer to an algorithm of high efficiency and arbitrary precision provided by Clamond and Dutykh [70]. A zeropenetration Neumann boundary condition $\partial \varphi / \partial n=0$ is imposed on $\partial \Omega_{\text {left }}, \partial \Omega_{\text {right }}$ and $\partial \Omega_{\text {seabed }}$. The first 20 Fourier components are included in this study.

In the wave-making zone, a relaxation is performed after each time-integration of the kinematic and dynamic free-surface boundary conditions by using the $4^{\text {th }}$-order explicit Runge-Kutta (RK4) scheme. The relaxation method is one of the most widely employed techniques for wave generation 


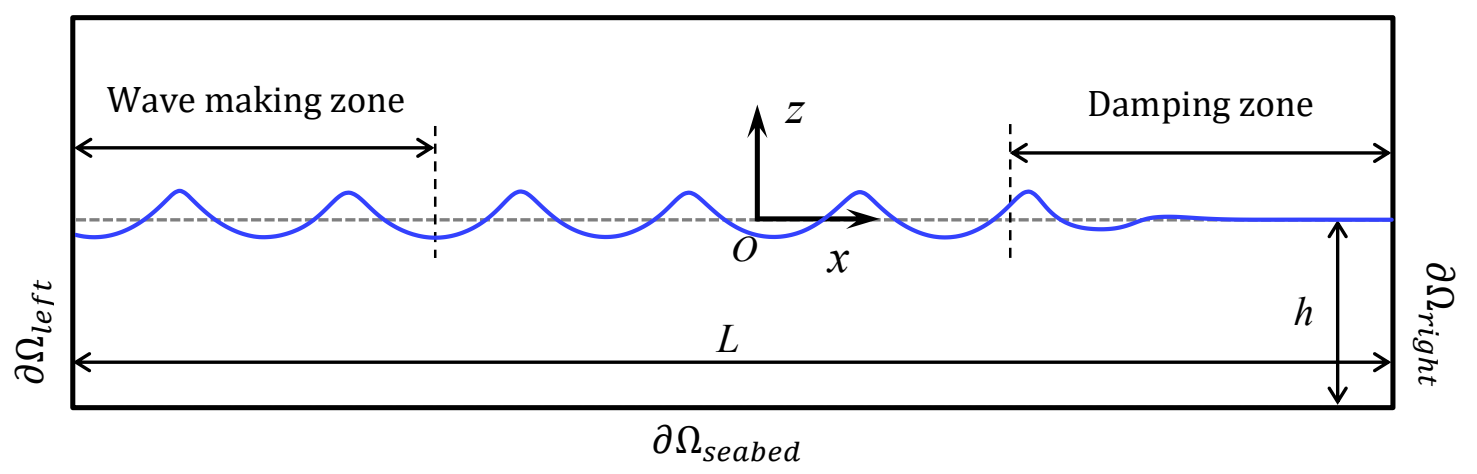

Figure 20: Sketch of a numerical wave tank.

Table 3: Definition of periodic waves.

\begin{tabular}{ccccccc}
\hline & $k a$ & $H(\mathrm{~m})$ & $T(\mathrm{~s})$ & $k\left(\mathrm{~m}^{-1}\right)$ & level $_{\max }$ & $C F L$ \\
\hline Case-1 & 0.1 & 0.0828 & 1.284 & 2.417 & $1|2| 3$ & 0.5 \\
Case-2 & 0.2 & 0.1660 & 1.265 & 2.417 & 2 & $0.3|0.6| 0.9$ \\
Case-3 & 0.3 & 0.2480 & 1.234 & 2.417 & $1|2| 3$ & 0.5 \\
Case-4 & 0.4 & 0.3310 & 1.192 & 2.417 & 2 & $0.3|0.6| 0.9$ \\
\hline
\end{tabular}

or absorption since it is very flexible and straightforward to implement $[3,71,72,73,74]$. After relaxation, the free-surface elevation and velocity potential on the free surface are given as:

$$
\begin{aligned}
\eta_{\text {new }}(x, t) & =\gamma_{r}(x) \eta_{\text {ref }}(x, t)+\left[1-\gamma_{r}(x)\right] \eta_{\text {num }}(x, t), \\
\varphi_{m, \text { new }}(x, t) & =\gamma_{r}(x) \varphi_{m, r e f}(x, t)+\left[1-\gamma_{r}(x)\right] \varphi_{m, \text { num }}(x, t),
\end{aligned}
$$

where, $\eta_{\text {num }}$ and $\eta_{\text {ref }}$ are the numerical result from time-integration and the reference solution for the wave elevation, respectively, while $\varphi_{m, n u m}$ and $\varphi_{m, r e f}$ represent the numerical and the reference velocity potentials. One of the most prevalent relaxation functions $\gamma_{r}(x)$ is proposed by Fuhrman et al. [72] as

$$
\gamma_{r}(x)= \begin{cases}\frac{\exp \left(\left(1-x_{r}\right)^{3.5}\right)-1}{\exp (1)-1}, & x-x_{0} \leq L_{W M Z} \\ 0, & x-x_{0}>L_{W M Z}\end{cases}
$$

where $x_{r}=\left(x-x_{0}\right) / L_{W M Z}$ is the local coordinate within the wave making zone. $x_{0}$ corresponds to the position of $\Omega_{\text {left }}$.

Four regular waves with different steepness shown in Table 3 are considered, where $H$ is the wave height, $T$ is the wave period, $k$ is the wave number and $k a$ represents the non-linearity of the waves, with $a=H / 2$. The $C F L$ number is defined as $C F L=c \Delta t / \Delta x$, where $c$ is the wave phase velocity, $\Delta t$ is time step and $\Delta x$ the grid spacing in the deepest grid level. In Table 3, the steepest wave with $k a=0.4$ is quite close to the deep-water breaking wave limit.

In order to test the performance of the wave tank, the wave elevation for $k a=0.1 \sim 0.4$ are plotted together with the stream-function solution at the time instant $t=20 \mathrm{~s}$ in Fig. 21. In Table 3, Case- 1 and Case- 3 are used to investigate the influence of grid refinement on the numerical solutions, 


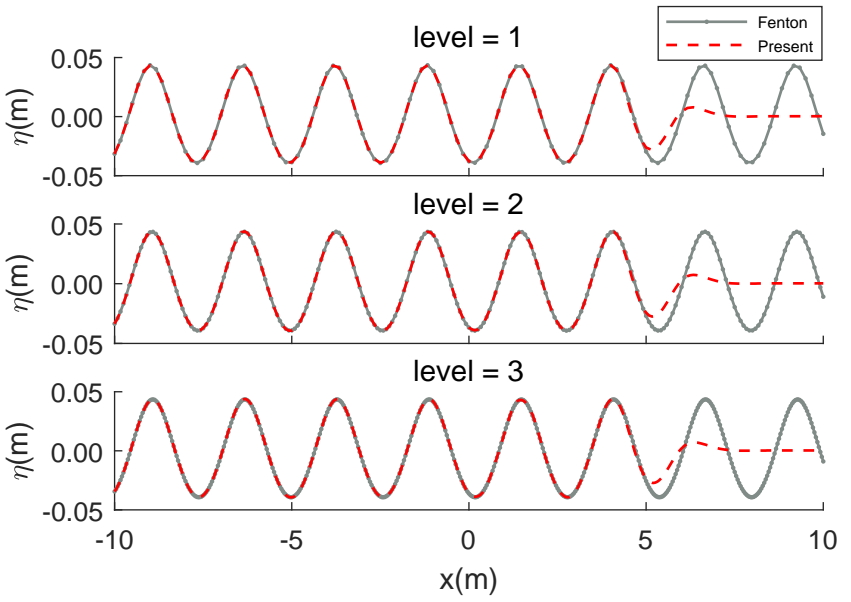

(a) $k a=0.1, C F L=0.5$

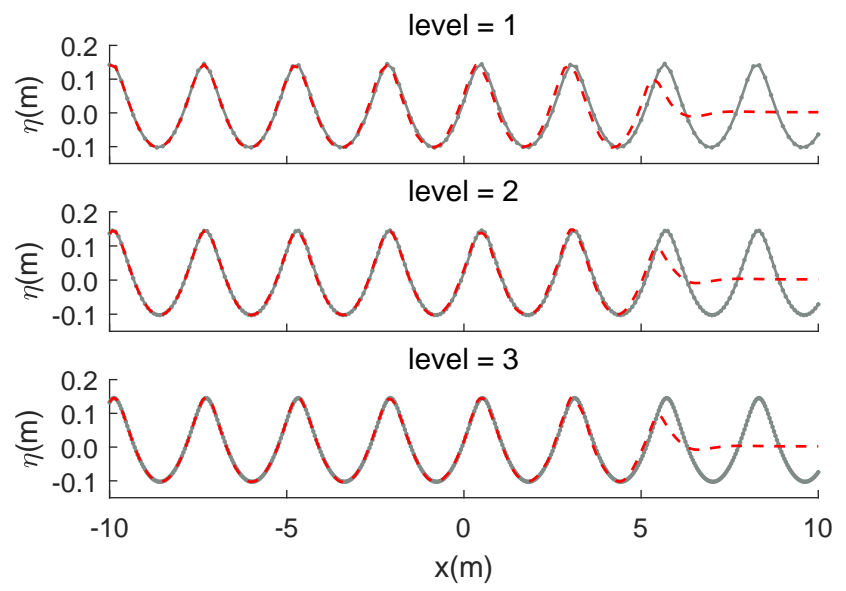

(c) $k a=0.3, C F L=0.5$

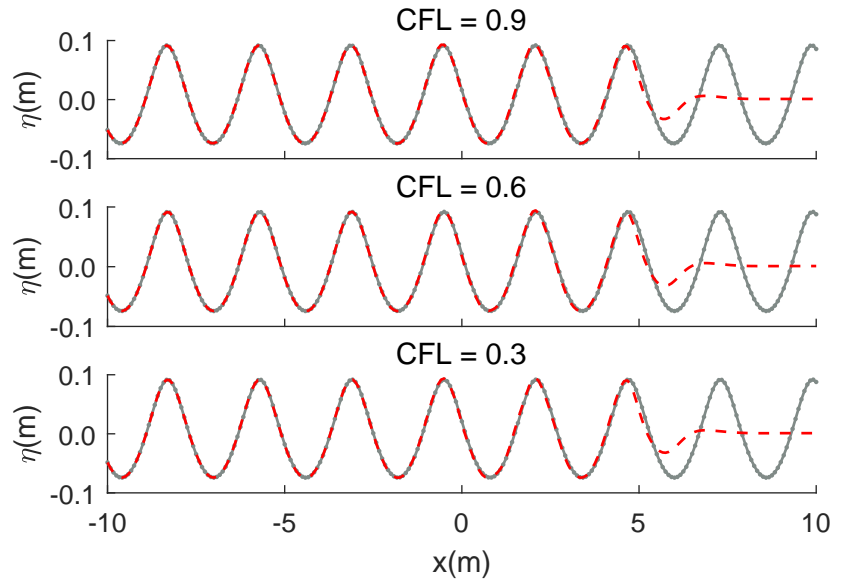

(b) $k a=0.2$, level $=2$

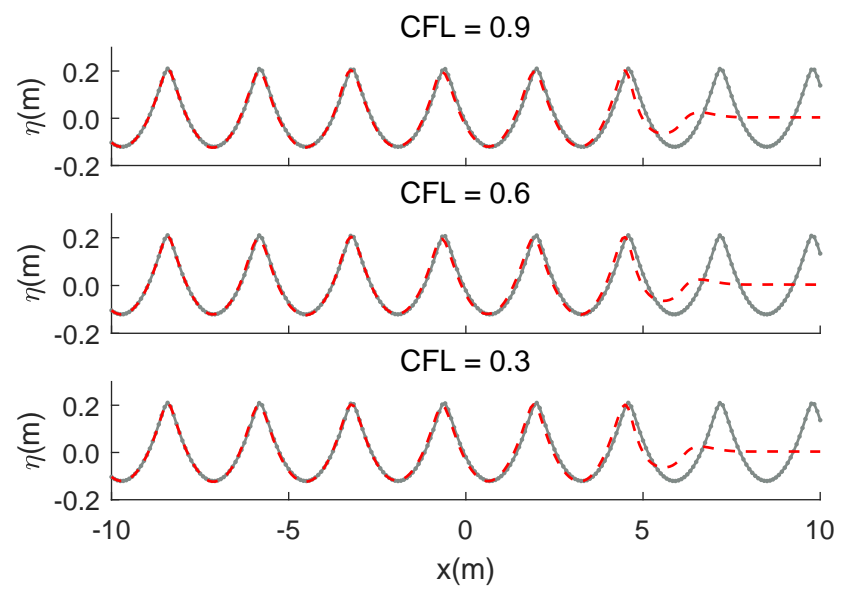

(d) $k a=0.4$, level $=2$

Figure 21: Wave profile for waves in Table 3. Present numerical results are compared with analytical solution provided by Rienecker and Fenton [68].

while Case-2 and Case- 4 are designed to study the influence of different time steps.

As shown in Fig. 21(a), there is an excellent agreement between the numerical and reference solutions outside of the damping zone for $k a=0.1$ with different grid refinements. As the wave steepness increases to $k a=0.3$, we notice an obvious phase lag for a level-1 mesh, due to the dispersion error introduced by the numerical solution. A finer grid, e.g. level $_{\max }=2$ or 3 , can significantly reduce the dispersion error. In general, the moderate grid refinement $\left(\right.$ level $\left._{\max }=2\right)$ with 32 nodes per wave length is sufficient to provide an accurate simulation for wave steepness up to $k a=0.3$. If we fix the grid spacing and use leve $_{\max }=2$ for Case- 2 and Case- 4 , it is seen from Fig. 21(b) and (d) that all time steps are sufficient as long as $C F L \leq 1$ thanks to the $4^{\text {th }}$-order timeintegration method. Strong nonlinearity of waves increases the challenge of computational modeling, and that is why the dispersion error looks more evident for Case- 4 with moderate mesh resolution (i.e. level $_{\max }=2$ ) than for the other cases. Therefore the Savitsky-Golay (SG) filter [75] with 13 points and $10^{\text {th }}$ order is applied to smooth the wave elevation and the velocity potential at each time step. It should be noted that the SG filter is only used for Case-4, not for the other cases in Table 3. 


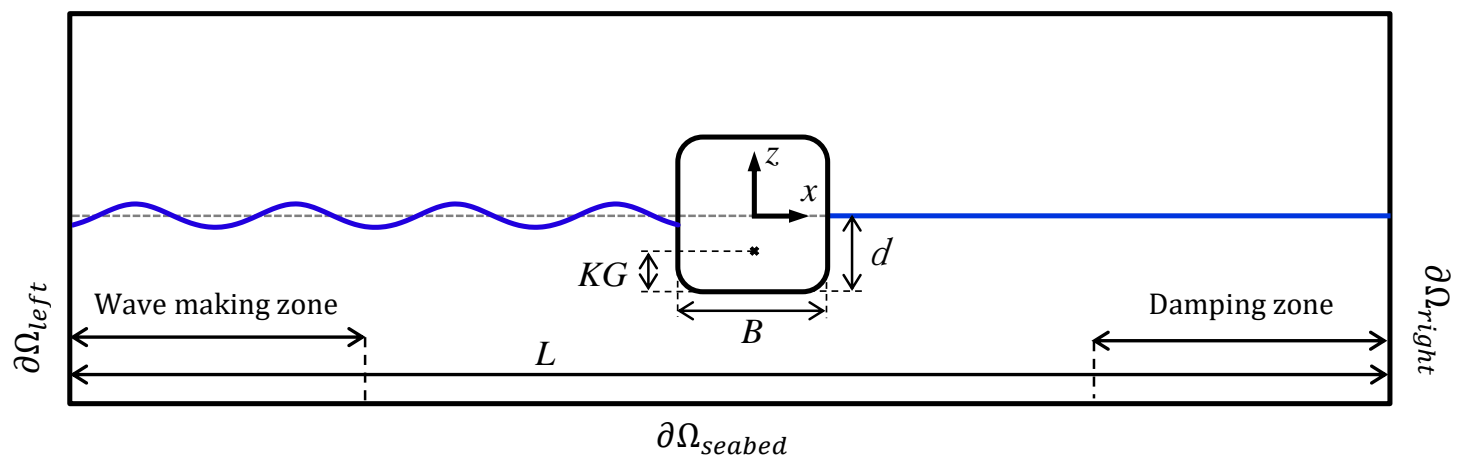

Figure 22: Sketch of a fixed semi-submerged barge in regular waves.

\section{- Fory 6.2 Fixed barge section in regular waves}

The developed 2D fully-nonlinear numerical wave tank is utilized to analyze a wave-diffraction problem, with a fixed surface-piercing barge in regular waves with various wave heights and frequencies. The wave loads on the barge were studied theoretically by Maruo [76], experimentally by Nojiri [77] and by Koo [78], Tanizawa [79] and Hanssen [47] using fully-nonlinear potential flow analyses. Fig. 22 shows the layout of the numerical wave tank. The length of the tank is set to be $L=8 L_{w}$ and the water depth is $h=L_{w}$, where $L_{w}$ is the wavelength. Waves are generated by the wave making zone described in $\S 6.1$. It also acts as a damping zone, absorbing the reflected waves from the barge, which avoids undesirable wave reflection back and forth between the left boundary and the barge. The length of both the wave making zone and the wave damping zone are taken as $L_{W M Z}=L_{W D Z}=2 L_{w}$. The center of this barge is coincident with the origin of a global Cartesian coordinate system $O x z$, with the origin at middle of the tank, $x$-axis pointing positively along the direction of wave propagating and $z$-axis pointing vertically upwards. The characteristic geometric parameters of the barge are chosen the same as the experiment from [77], with a breadth $B=0.5 \mathrm{~m}$, a draught $d=B / 2$, a round corner radius $r=0.064 \mathrm{~m}$ and a gravitational height $K G=0.135 \mathrm{~m}$.

As in [79], incident regular waves with two different wave heights $H=0.01 \mathrm{~m}$ and $H=0.07$ $\mathrm{m}$ are used. To generate the waves in the wave making zone, we have used both linear waves and nonlinear stream-function incident waves as used in $\S 6.1$ as inputs in Eq. (42). In case a linear incident wave solution is used, the generated wave outside of the wave making zone will still develop into a fully-nonlinear wave. The non-dimensional wave frequency $\xi_{B}=\omega^{2} B / 2 g$ ranging from 0.25 to 2.00 are simulated, where $\omega$ is angular wave frequency and $g$ denotes gravitational acceleration. The wave frequencies and other important parameters, including the wave lengths $L_{w}^{l}$ and $L_{w}^{s f}$ calculated by a linear wave theory and stream function theory respectively, the wave period $T$ and the wave steepness $k a$, where $k$ is the wave number and $a$ is the wave amplitude defined as $a=H / 2$, are listed in Table 4. For all cases in Table 4, a time step $T / \Delta t$ from 40 to 100 is used from the lowest to the highest frequencies. The grid spacing at the deepest grid level is subject to the criterion $d x=d z=\min \left(B / 14, L_{w} / 30\right)$. The ramp time for wave making is set to be $t_{\text {ramp }}=4 T$. Wave markers here will be subjected to the fully Lagrangian free-surface kinematic and dynamic boundary conditions, uniformly distributed along the $x$-axis. The explicit RK4 time scheme is used to integrate the Lagrangian time derivative of the positions and velocity potential of wave markers.

The time history of non-dimensional forces along the $x$-axis (sway force) and $z$-axis (heave force) and the moment around the $y$-axis (roll moment) on the barge section for linear waves with wave 
Table 4: Definition of periodic waves for a fixed barge in waves.

\begin{tabular}{ccccccc}
\hline$\xi_{B}$ & $\omega\left(\mathrm{s}^{-1}\right)$ & $L_{w}^{l}(\mathrm{~m})$ & $L_{w}^{s f}(\mathrm{~m})$ & $T(\mathrm{~s})$ & $k a_{H=1 \mathrm{~cm}}$ & $k a_{H=7 \mathrm{~cm}}$ \\
\hline 0.250 & 3.132 & 6.283 & 6.288 & 2.006 & 0.005 & 0.035 \\
0.500 & 4.429 & 3.142 & 3.159 & 1.419 & 0.010 & 0.070 \\
0.550 & 4.645 & 2.856 & 2.874 & 1.353 & 0.011 & 0.077 \\
0.600 & 4.851 & 2.618 & 2.638 & 1.295 & 0.012 & 0.084 \\
0.650 & 5.049 & 2.417 & 2.439 & 1.244 & 0.013 & 0.091 \\
0.700 & 5.240 & 2.244 & 2.270 & 1.199 & 0.014 & 0.098 \\
0.750 & 5.424 & 2.094 & 2.118 & 1.158 & 0.015 & 0.105 \\
1.000 & 6.263 & 1.571 & 1.601 & 1.003 & 0.020 & 0.140 \\
1.250 & 7.002 & 1.257 & 1.294 & 0.897 & 0.025 & 0.175 \\
1.500 & 7.671 & 1.047 & 1.091 & 0.819 & 0.030 & 0.210 \\
1.750 & 8.285 & 0.898 & 0.948 & 0.758 & 0.035 & 0.245 \\
2.000 & 8.857 & 0.785 & 0.841 & 0.709 & 0.040 & 0.280
\end{tabular}

height $H=0.01 \mathrm{~m}$ and $H=0.07 \mathrm{~m}$ are shown in Fig. 23, where non-dimensional frequencies $\xi_{B}=$ $(0.5,1.0,1.5)$ are chosen to display. Only 20 wave periods are shown since the simulation reaches steady state after approximate $t / T=10$. Nonlinear features clearly increase as the incident wave frequency grows, particularly for the heave force in short waves. We can see from Table 4 that the largest wave steepness is $k a=0.28$. When this incident wave reaches the barge, partially standing waves are expected on the weather side of the barge, in particular for shorter incident waves due to stronger wave diffraction, leading to higher nonlinearities. In order to suppress numerical instabilities, a non-centered selective filter is applied near the wave-body intersection waterline [80], while a 13points and $10^{\text {th }}$-order SG filter is again used for the free surface away from the body $[75,81]$. This combination of mixed filters is also used for other wave-body intersection problems in the following applications.

A Fourier analysis of the time series of the load $F(t)$ on the barge is conducted as

$$
F(t)=a_{0}+\sum_{n=1}^{N}\left(a_{n} \cos (n \omega t)+b_{n} \sin (n \omega t)\right)
$$

where $a_{n}$ and $b_{n}$ can be calculated by a least squares fit to a steady-state portion of the time-series. Here the $n$-th order load is calculated as $F^{(n \omega)}=\sqrt{a_{n}^{2}+b_{n}^{2}}$ and $a_{0}$ denotes the mean wave load. In the present study, the last 5 periods of the time histories are taken to perform the Fourier analysis. Fig. 24 shows the normalized sway mean-drift force $F_{x}^{(0)}$, the first harmonics of the sway force $F_{x}^{(1 \omega)}$, heave force $F_{z}^{(1 \omega)}$ as well as roll moment $M_{y}^{(1 \omega)}$ on the fixed barge. The present results of linear waves with wave height $H=0.01 \mathrm{~m}$ and $H=0.07 \mathrm{~m}$ and nonlinear stream-function waves with wave height $H=0.07 \mathrm{~m}$ are compared with theoretical solution from [76] based on a perturbation method, experimental results of [77] and fully-nonlinear numerical analysis of $H=0.07 \mathrm{~m}$ from [79] using linear waves as input signal. Note that all the wave forces are obtained through direct pressure integration along the wetted surface of the barge. The present results are generally in good agreement with the analytical solutions, numerical results of [79] and experimental results, despite some deviations at high frequencies from the experiments. The force results by the linear wave theory and the stream function theory are compared in Fig. 24. Although the input wave signals are 


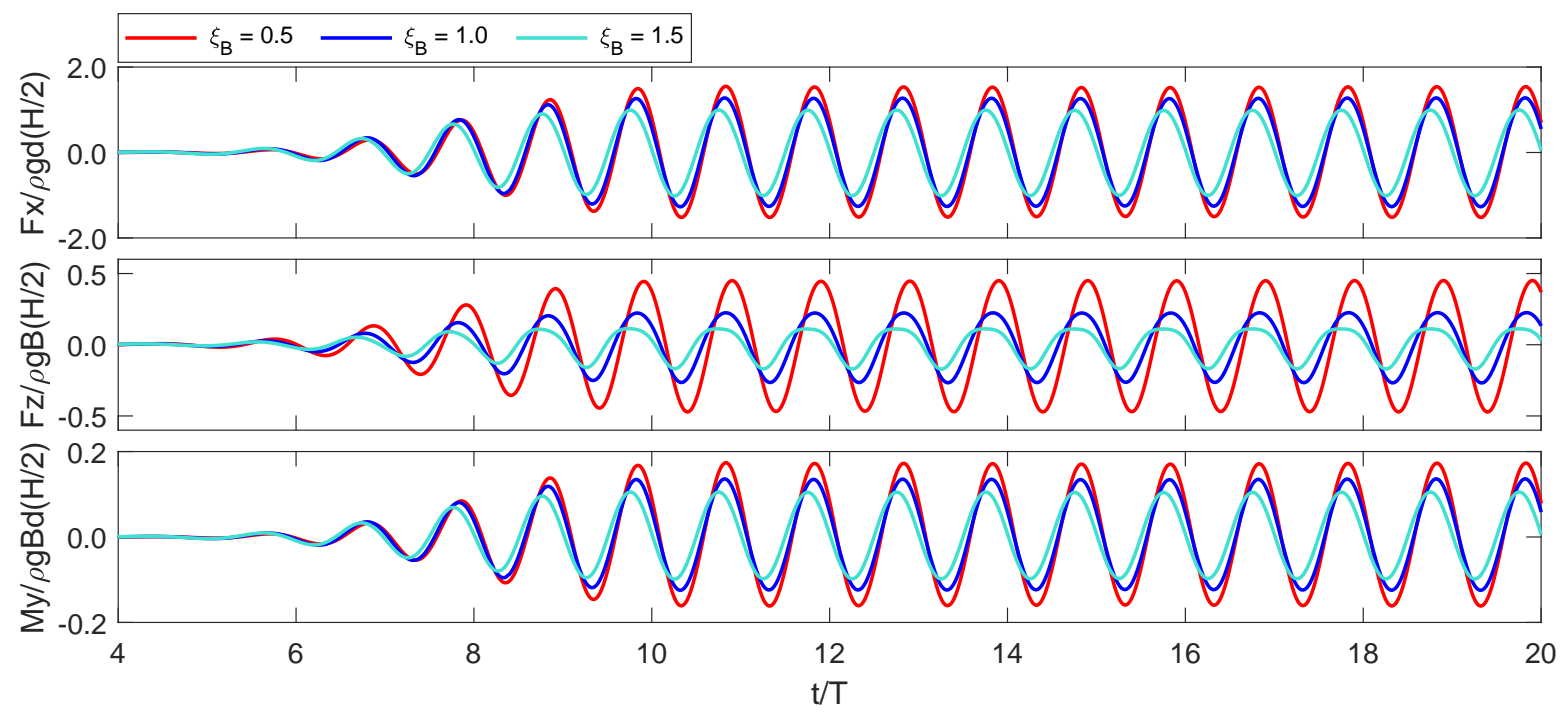

(a) Wave height $H=0.01 \mathrm{~m}$

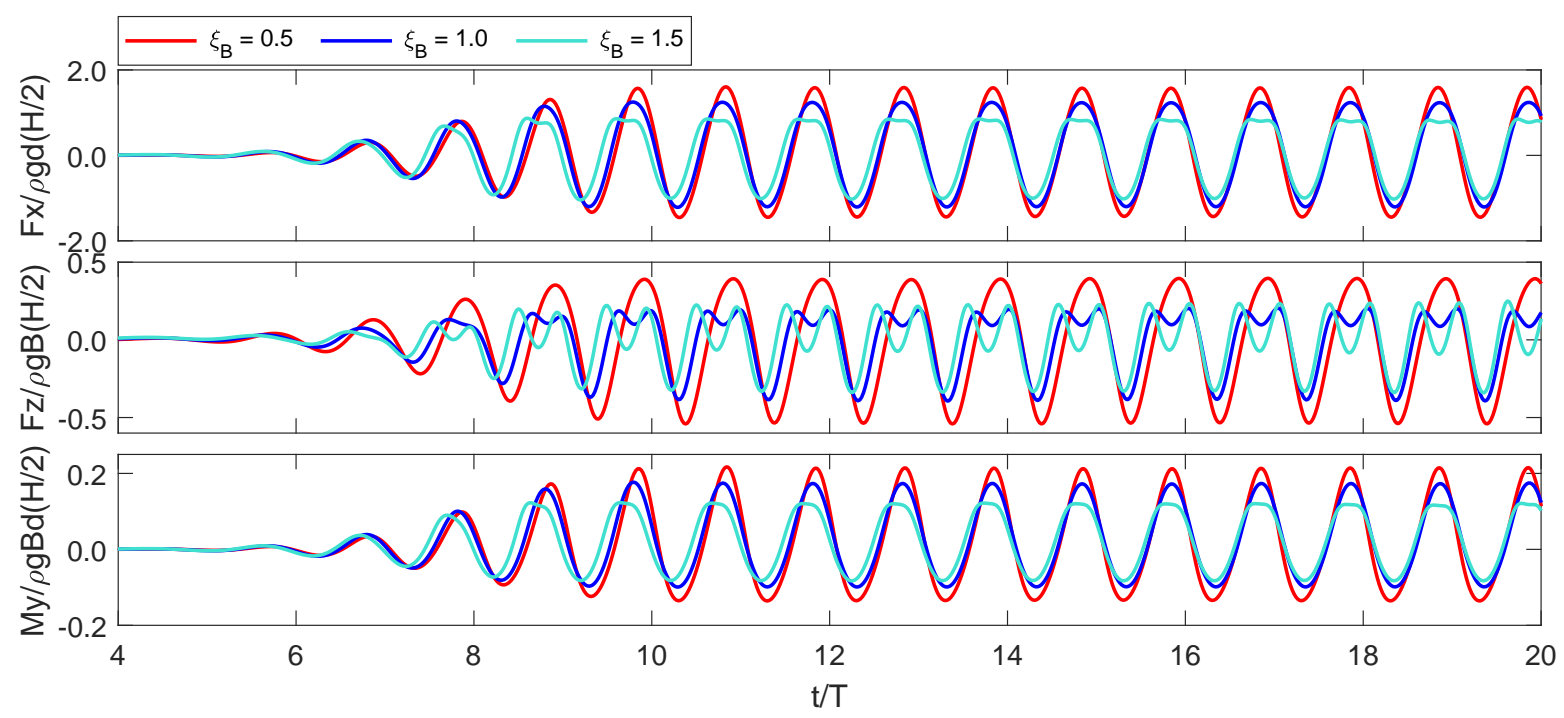

(b) Wave height $H=0.07 \mathrm{~m}$

C Figure 23: Non-dimensional force history for a fixed semi-submerged barge in waves by linear wave theory for wave height $H=0.01 \mathrm{~m}$ and $H=0.07 \mathrm{~m}$ with non-dimensional wave frequency $\xi_{B}=0.5$, $1.0,1.5$.

different, the difference of the results by linear and nonlinear waves with $H=0.07 \mathrm{~m}$ is not obvious, except for the mean sway drift force at frequency $\xi_{B}=2.0$. When the stream function theory is applied in the wave making zone, our numerical results tend to share a similar tendency as that in the experiment [77], i.e. the non-dimensional mean sway drift force $F_{x}^{(0)}$ tend to decrease as the frequency increases. The deviation from the theoretical solution may result from the strong nonlinearity of waves at higher frequencies. Since a fixed wave height is considered for all frequencies, the non-linearity increases with frequency, which can be understood from the $k a$ values in Table 4. In the low frequency region, both the results of $H=0.01 \mathrm{~m}$ and $H=0.07 \mathrm{~m}$ for $F_{x}^{(0)}, F_{x}^{(1 \omega)}$ and $F_{z}^{(1 \omega)}$ are very close to each other. 


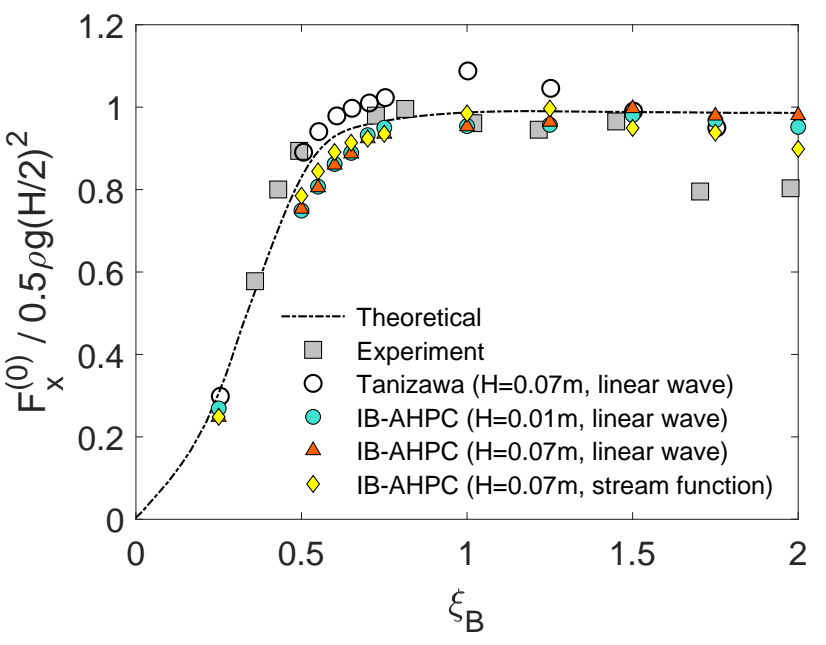

(a) Mean sway drift force

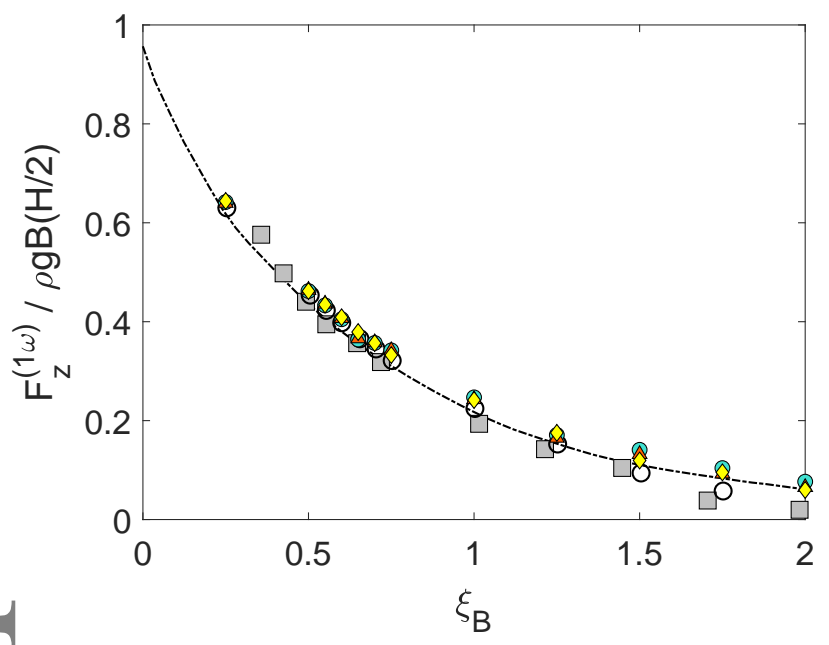

(c) Heave force

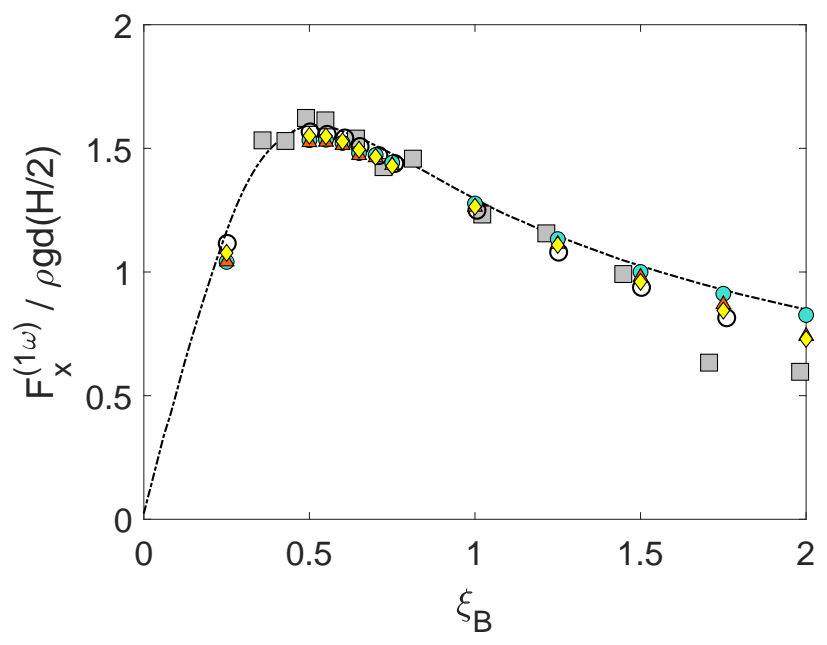

(b) Sway force

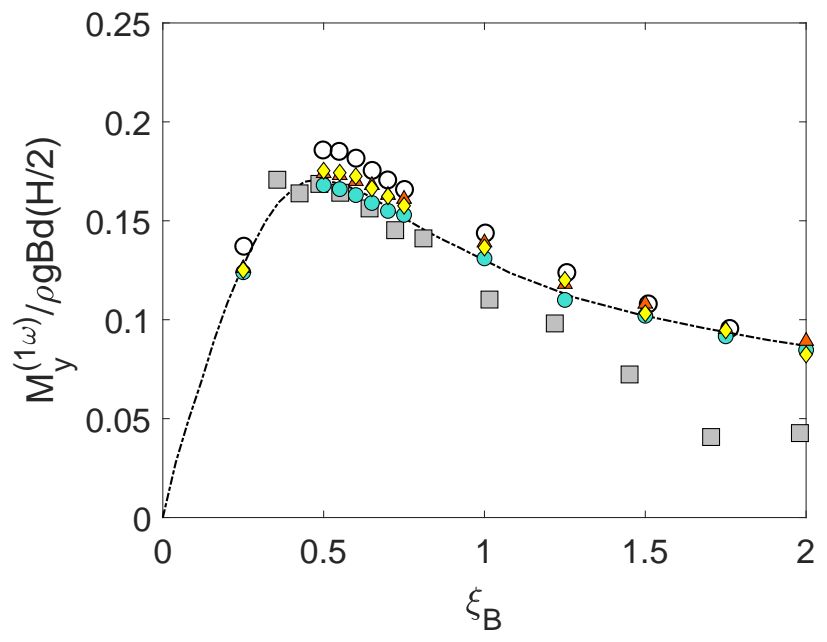

(d) Roll moment

Figure 24: Normalized first harmonic component wave exciting loads and the mean sway drift force on the fixed barge. Present numerical results by linear waves $(H=0.01 \mathrm{~m}$ and $H=0.07 \mathrm{~m})$ and nonlinear waves by the stream function theory $(H=0.07 \mathrm{~m})$ are compared with theoretical linear solution from [76], experimental solution from [77] and numerical results from [79].

\subsection{Forced heaving of a $2 \mathrm{D}$ Lewis-form barge section in still water}

The radiation problem of a Lewis-form barge harmonically heaving on the free surface is considered here to test the capability of the numerical model on simulating the wave-body interaction. Fig. 25 gives a snapshot of an adaptive grid system with level $_{\max }=2$ and $e$-degree $=2$ for this heaving barge section in a numerical wave tank. Some colored nodes with different node types can be referred from $\S 3.2$ and $\S 4.2$. In this part, the breadth of the Lewis-form barge is kept the same as in $\S 6.2$ and the block coefficient of this Lewis-form section is defined as $\epsilon=S /(B d)=0.989$. The length of the wave tank is $L=8 L_{w}$ and the water depth here is $h=\max \left(L_{w}, 3 B\right)$. The wave making zone is changed into a damping zone and the length of the damping zone at left and right end of this tank is $L_{W D Z}=2 L_{w}$. The initial draught of the barge is also set to be 
Table 5: Definition of periodic waves generated by a harmonically heaving barge in undisturbed water. amplitude is defined as $\sigma_{\text {heave }}=\eta_{a} /(B / 2)=0.222$. In order to achieve a good accuracy close to the wave-body intersection point, we have used a grid size in the deepest level based on the criterion $d x=d z=\min \left(B / 25, L_{w} / 40\right)$. A time step of $T / \Delta t=40$ is chosen for all frequencies. The main properties of the waves generated by the heaving motion of the barge are summarized in Table 5, which are based on linear dispersion relationship. The ramp time to gradually start the motion is set as $t_{\text {ramp }}=4 T$ and the simulation time is $t_{\text {sim }}=10 T$ for each case listed in Table 5 . A Fourier analysis is performed on the time history of heaving force on the barge using Eq. (44). Then the added mass $A_{33}$ and damping coefficients $B_{33}$ can be found from the first harmonic components,

$$
A_{33}=-\frac{b_{3,1}-C_{z} \eta_{a}}{\omega^{2} \eta_{a}}, \quad B_{33}=\frac{a_{3,1}}{\omega \eta_{a}}
$$

where the hydrostatic restoring coefficient is given as $C_{33}=\rho g B$.

$d=B / 2$. The heave motion is given as $\eta_{\text {heave }}(t)=-\eta_{a} \sin \omega t$, and the non-dimensional heaving
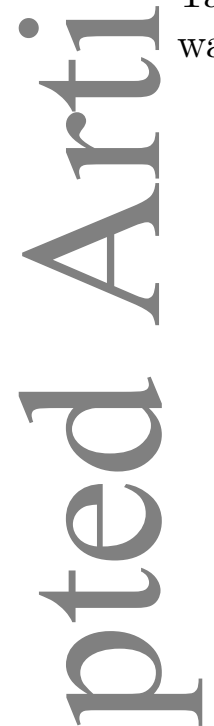

\begin{tabular}{ccccc}
\hline$\xi_{B}$ & $\omega\left(\mathrm{s}^{-1}\right)$ & $L_{w}(\mathrm{~m})$ & $T(\mathrm{~s})$ & $h(\mathrm{~m})$ \\
\hline 0.250 & 3.132 & 6.283 & 2.006 & 6.283 \\
0.500 & 4.429 & 3.142 & 1.419 & 3.142 \\
0.750 & 5.424 & 2.094 & 1.158 & 2.094 \\
1.000 & 6.263 & 1.571 & 1.003 & 1.571 \\
1.200 & 6.862 & 1.309 & 0.916 & 1.500 \\
1.500 & 7.671 & 1.047 & 0.819 & 1.500 \\
1.750 & 8.285 & 0.898 & 0.758 & 1.500 \\
2.000 & 8.857 & 0.785 & 0.709 & 1.500
\end{tabular}

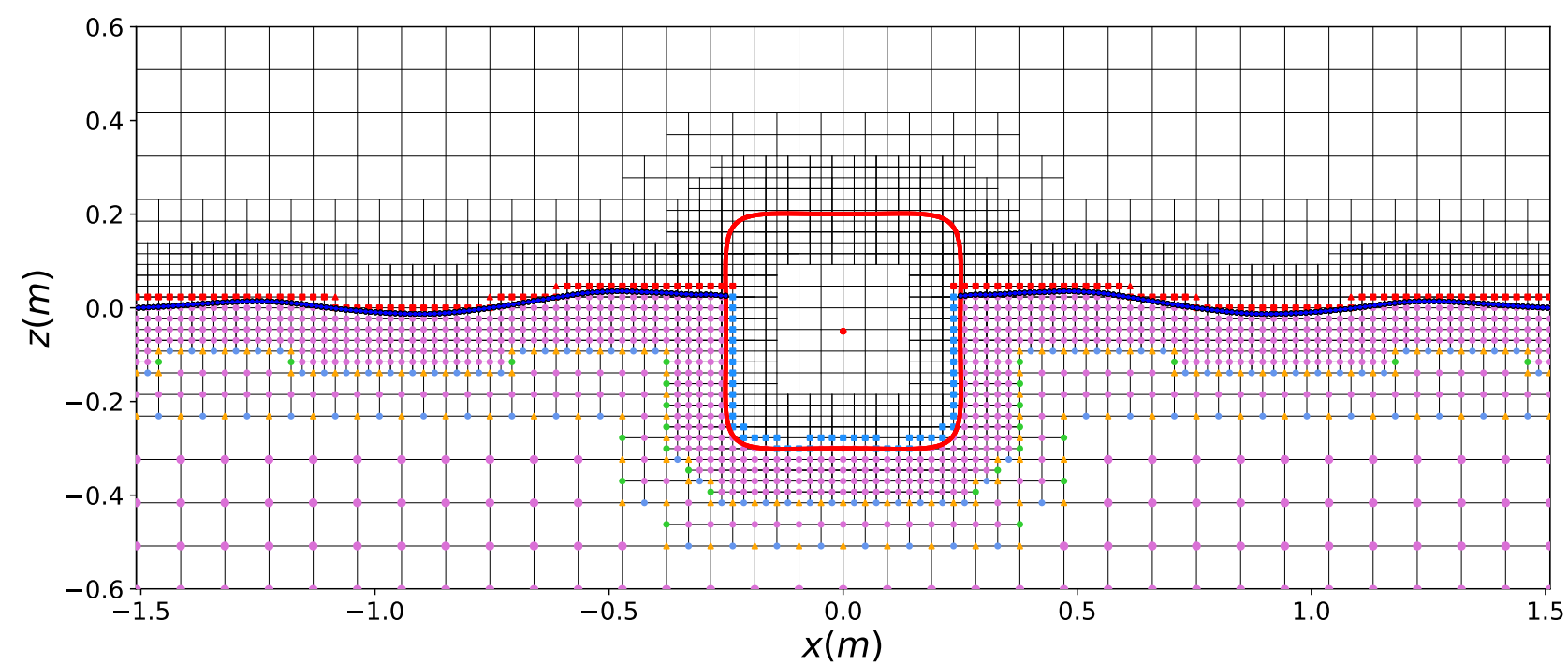

Figure 25: Adaptive Cartesian grid system for a forced heaving Lewis-form barge at free surface. level $_{\max }=2$ and $e$-degree $=2$ 

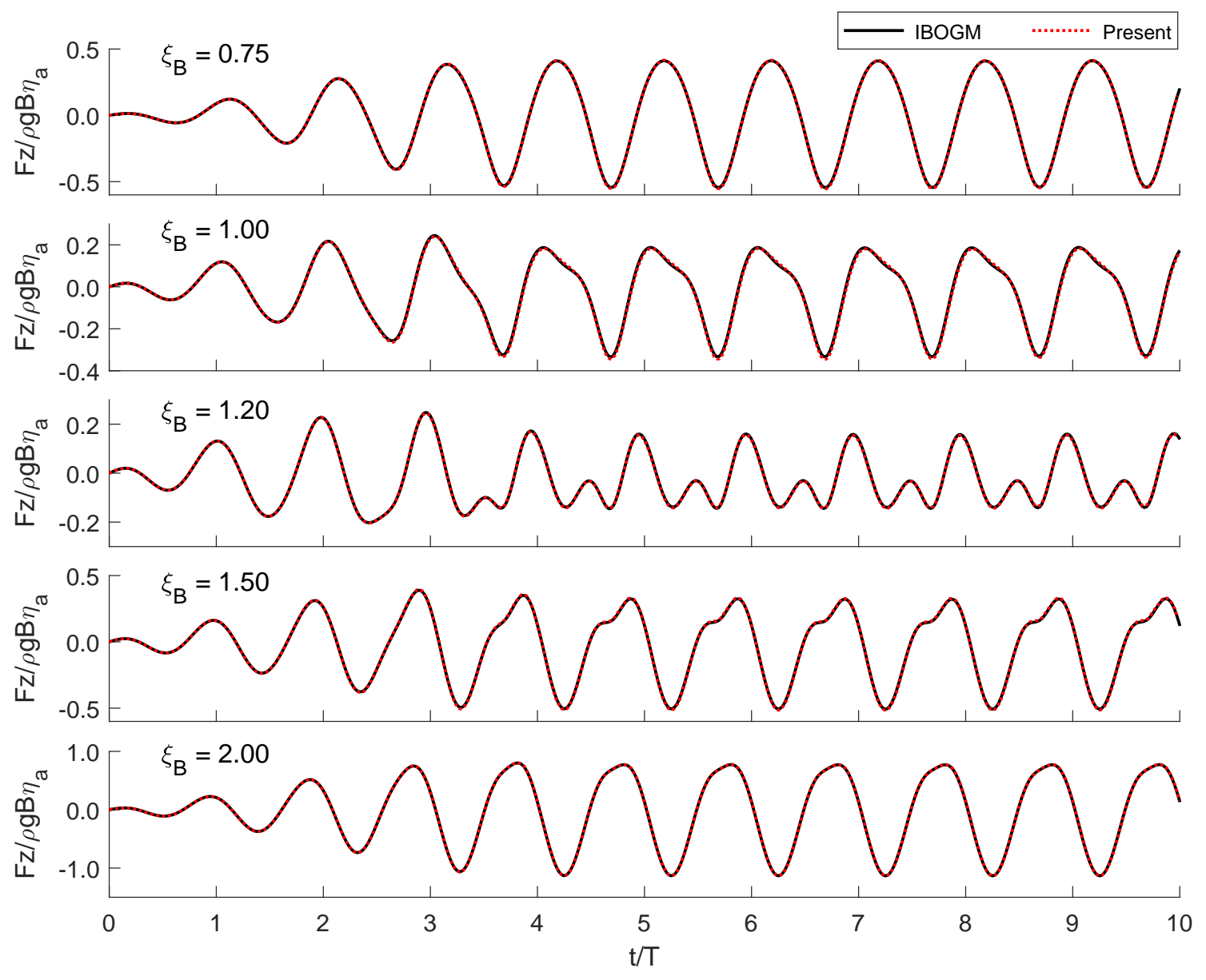

Figure 26: Time history of normalized heaving force (excluding restoring force) of a harmonic heaving barge for frequency $\xi_{B}=(0.75,1.00,1.20,1.50,2.00)$. Present work on time history of heaving force is compared with the numerical reference from the IBOGM in [47].

Fig. 26 shows the time history of normalized heaving forces on the barge for five different frequencies $\xi_{B}=(0.75,1.00,1.20,1.50,2.00)$. Our present results are compared with another fully-nonlinear HPC solver based on Immersed Boundary-Overlapping Grid Method (IBOGM) [47], providing reference results in the form of time series. The IBOGM has been carefully verified and validated as a highly accurate method in [51] and [47]. Based on direct comparison of time series, the present results and the IBOGM are visually in perfect agreement. The time series effectively prove that there are no spurious oscillations by applying the Lagrangian acceleration potential method (LAPM) described in $\S 3.3$ to evaluate $\partial \varphi / \partial t$. Fig. 27 and 28 respectively display the field distribution of the velocity potential $\varphi$ and the Lagrangian acceleration potential $\Psi$ at $t=\left(\frac{1}{4} T, \frac{2}{4} T, \frac{3}{4} T, T\right)$ for a Lewis-form barge section heaving at the free surface at a frequency $\xi_{B}=2.00$. It is clear to see the continuity of the field for both $\varphi$ and $\Psi$, which contributes to the smoothness of the force signals in Fig. 26 by applying the LAPM.

Using an IBM combined with a finite-difference method to estimate the time derivative of the velocity potential in analyses with moving bodies, inevitably leads to spurious pressure- and force oscillations [49]. Another way to evaluate $\varphi_{t}$ in the Bernoulli's equation is the Eulerian acceleration 


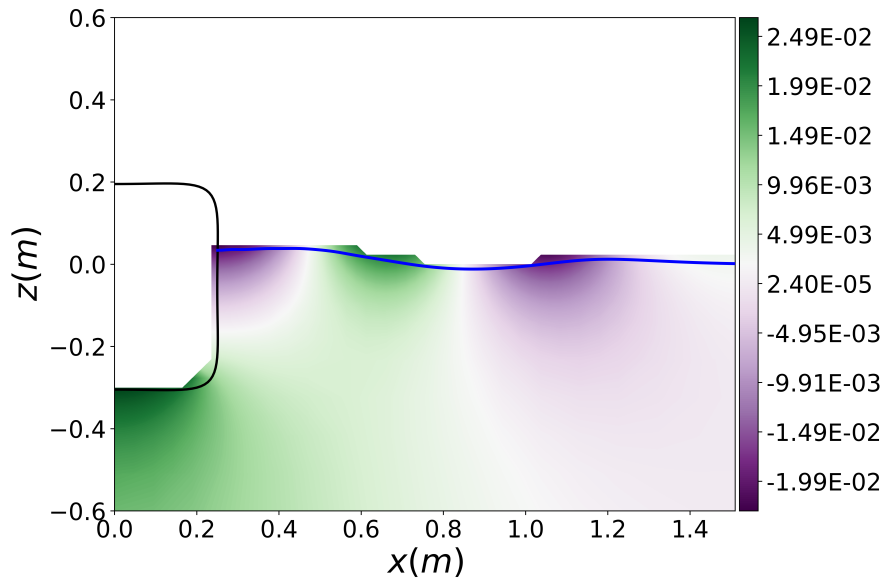

(a) $t=\frac{1}{4} T$

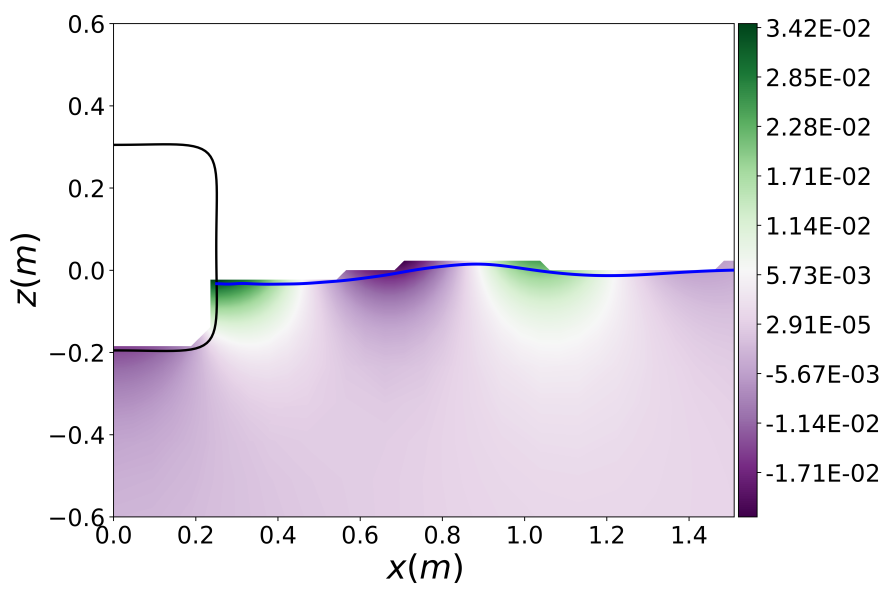

(c) $t=\frac{3}{4} T$

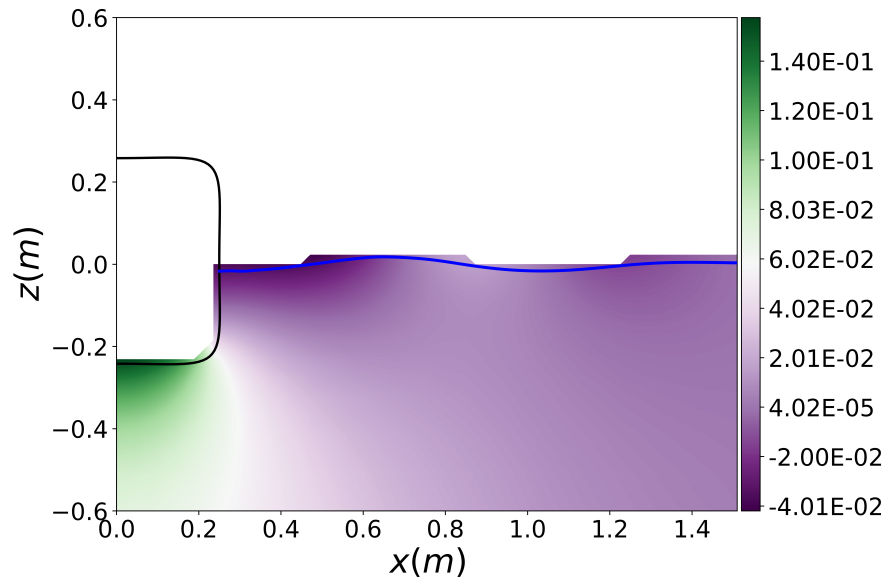

(b) $t=\frac{2}{4} T$

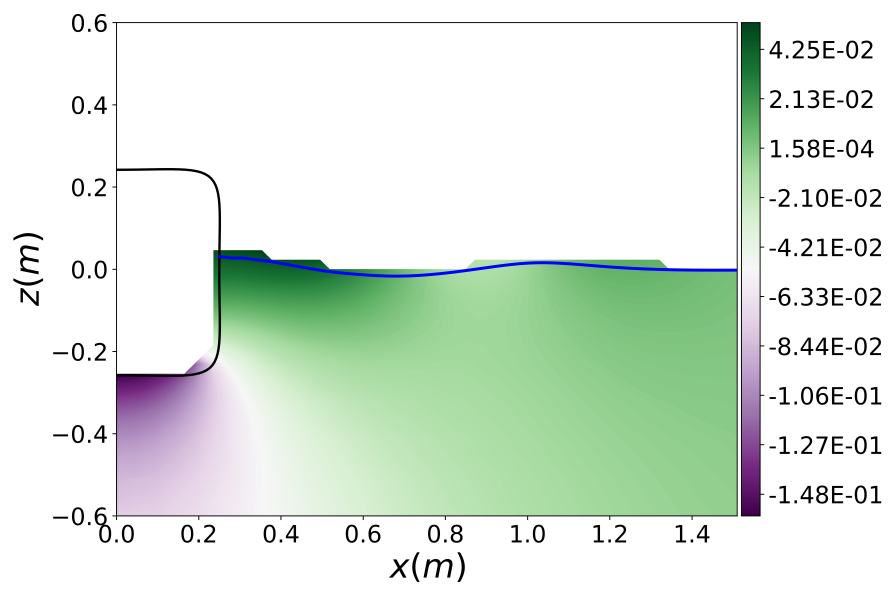

(d) $t=T$

| 1 igure 27: Field distribution of the velocity potential $\varphi$ at $t=\left(\frac{1}{4} T, \frac{2}{4} T, \frac{3}{4} T, T\right)$ for a Lewis-form barge harmonically heaving at free surface at the non-dimensional frequency $\xi_{B}=2.00$. level $_{\max }=2$ and $e$-degree $=2$.

780 potential method (EAPM). See, e.g. [59] and [58], and a short description in $\S 3.3$. In Fig. 29, the results of time history of heaving force for two selected frequencies $\xi_{B}=(1.20,2.00)$ by using the EAPM and LAPM in the present analysis are compared. Here, the grid refinement, using the EAPM and LAPM, for one specific frequency is kept the same. In our numerical experiments, we found that even if we apply the EAPM from [59], we still cannot get the time history of force as smooth as that by the LAPM. It also turns out that the EAPM performs worse for cases with stronger nonlinearities. Although the result from the EAPM tends to improve with weaker nonlinearities, it is still not as good as the LAPM. The difference can be explained by the second-order spatial derivative term of the velocity potential in the Neumann boundary conditions in Eq. (20) for the EAPM, which is challenging to estimate accurately, especially for where the flow changes strongly. The LAPM, however, does not involve any derivatives in the body boundary condition. See Eq. (25).

The present results of added mass and damping coefficients as well as $1^{\text {st }}$-order heave forces are compared with linear analytical solutions and experimental measurements from Tasai and Koterayama [82], fully-nonlinear BEM results of Grytøyr [83] and numerical solutions from IBOGM by 


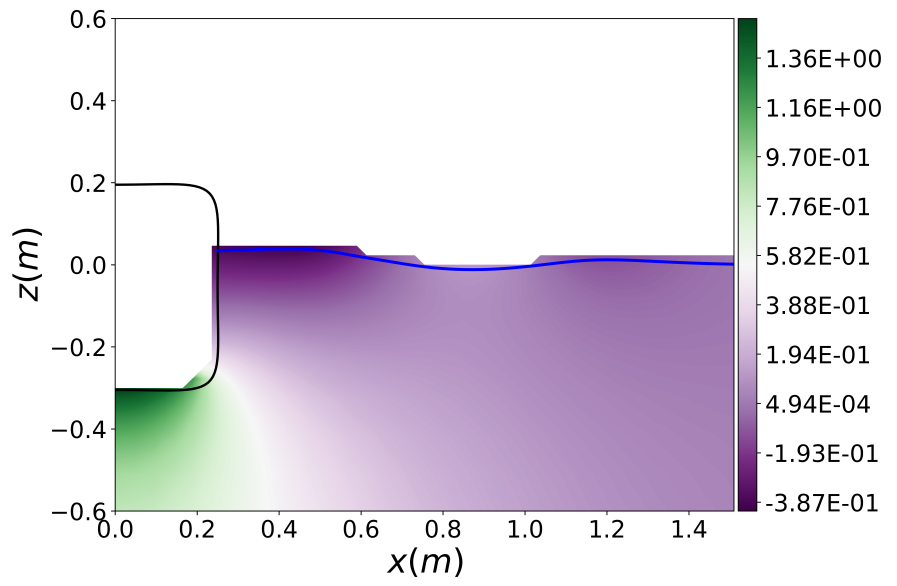

(a) $t=\frac{1}{4} T$

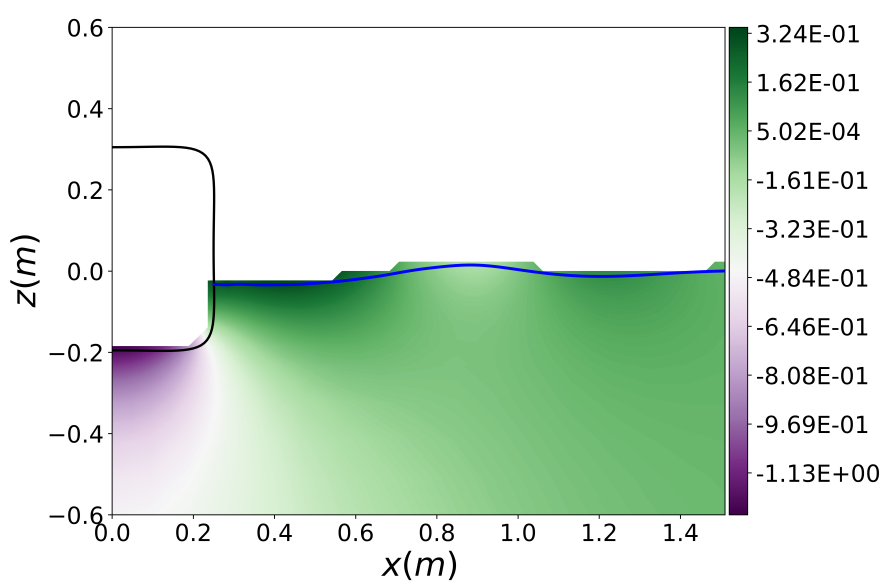

(c) $t=\frac{3}{4} T$

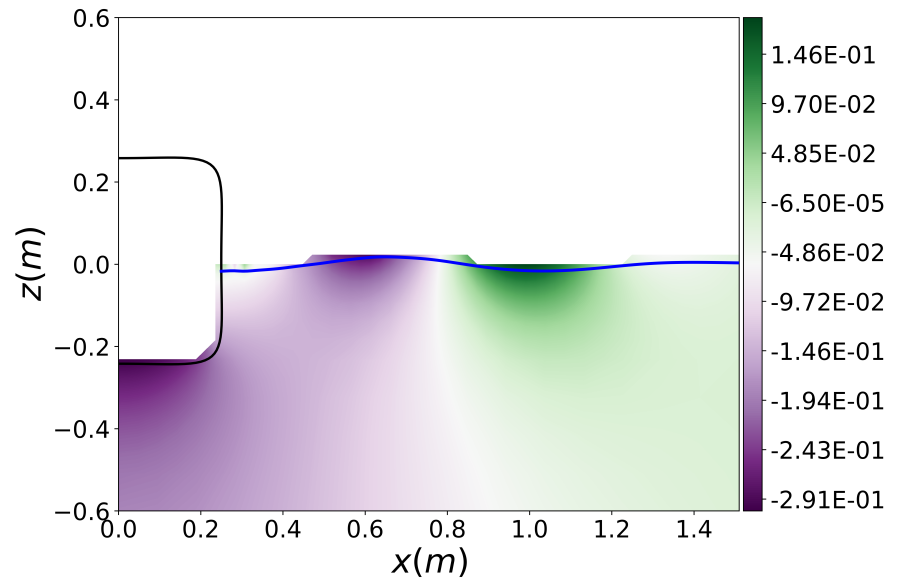

(b) $t=\frac{2}{4} T$

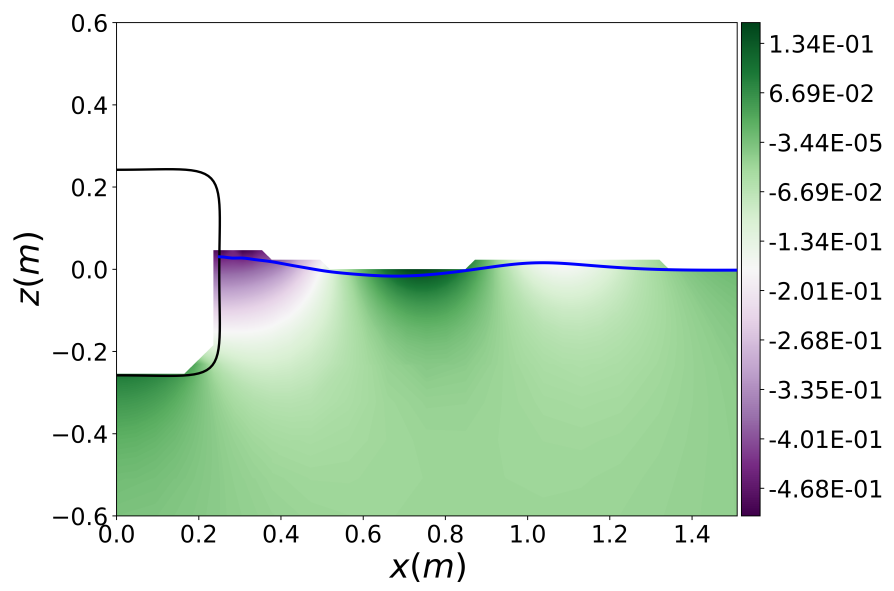

(d) $t=T$

|igure 28: Field distribution of the Lagrangian acceleration potential $\Psi$ at $t=\left(\frac{1}{4} T, \frac{2}{4} T, \frac{3}{4} T, T\right)$ for a Lewis-form barge harmonically heaving at free surface at the non-dimensional frequency $\xi_{B}=2.00$. level $_{\max }=2$ and $e$-degree $=2$.

794 Hanssen [47], as shown in Figure 30. The consistence are fairly satisfactory. For the $1^{\text {st }}$-order force, present results are pretty in agreement with the fully nonlinear results of [83] and [47]. Tasai and Koterayama [82] have also presented the $2^{\text {nd }}$-order force component in two different ways: the normalized force amplitudes and the ratio of the $2^{\text {nd }}$-order to the $1^{\text {st }}$-order force amplitudes. They also gave the ratio for the $3^{\text {rd }}$-order to the $1^{\text {st }}$-order force amplitudes, but no normalized experimental results of the $3^{r d}$-order force were presented. Here, our present numerical results of higher order force components are compared with experimental results of [82], fully-nonlinear BEM results of [83] and numerical results of IBOGM from [47], as shown in Fig. 31. We can notice that, despite of slightly deviations comparing to the BEM and the IBOGM for the $2^{n d}$ - and $3^{r d}$-order force in the high frequency region, generally speaking, the present results match well with reference solutions. It is not clear how large uncertainties are in the experiments, in particular for the $3^{\text {rd }}$-order forces at high frequencies, which is known to be challenging to measure accurately in model-scale experiments. The experimental results show that the $3^{\text {rd }}$ force component is much larger than the $2^{\text {nd }}$-order component at $\xi_{B}=1.8$, while none of the presented numerical results are able to reproduce this. 

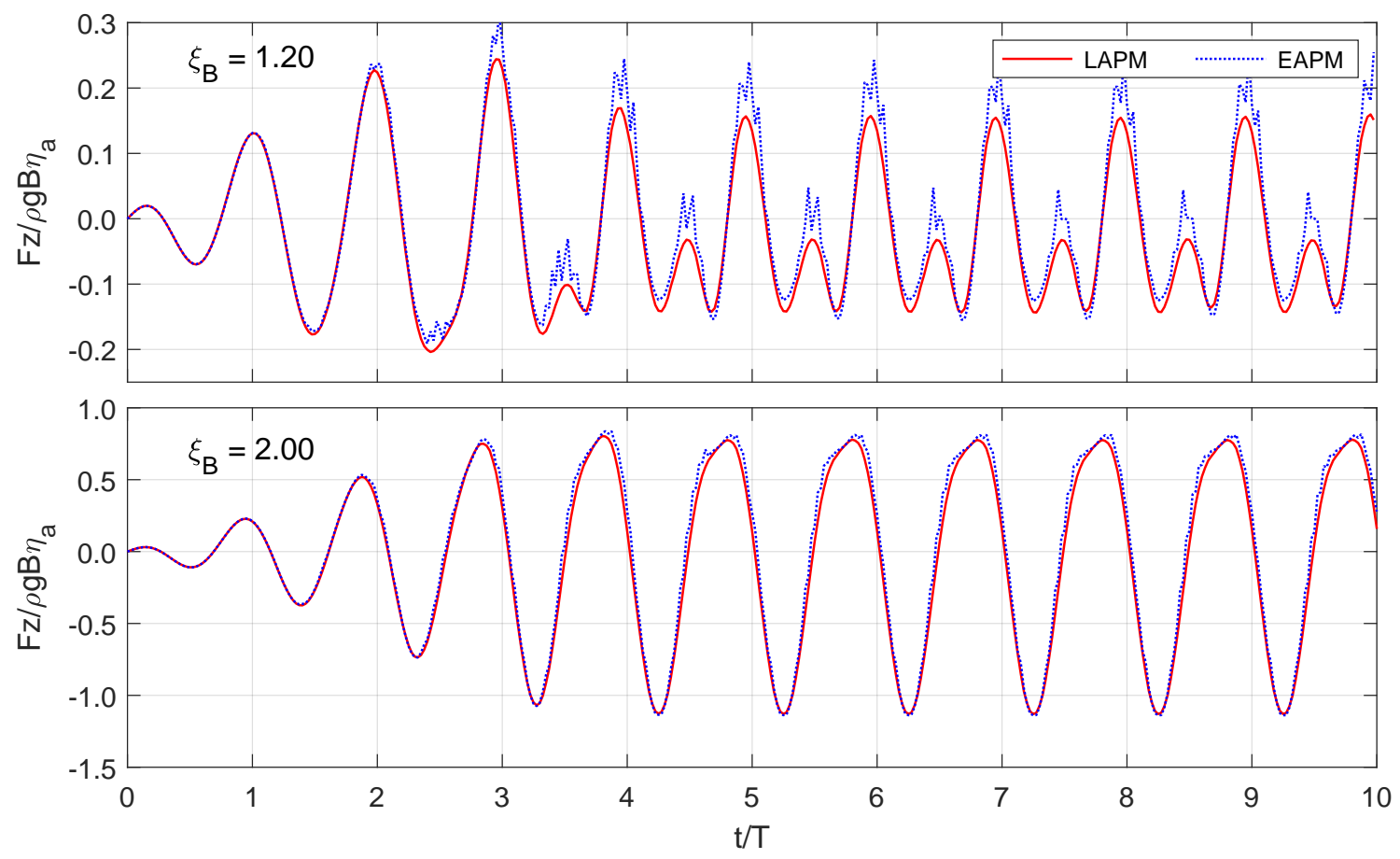

Figure 29: Time history of normalized heaving force (excluding restoring force) of a harmonic heaving barge for frequency $\xi_{B}=(1.20,2.00)$, using the EAPM and LAPM, respectively, to estimate the time derivative of the velocity potential.
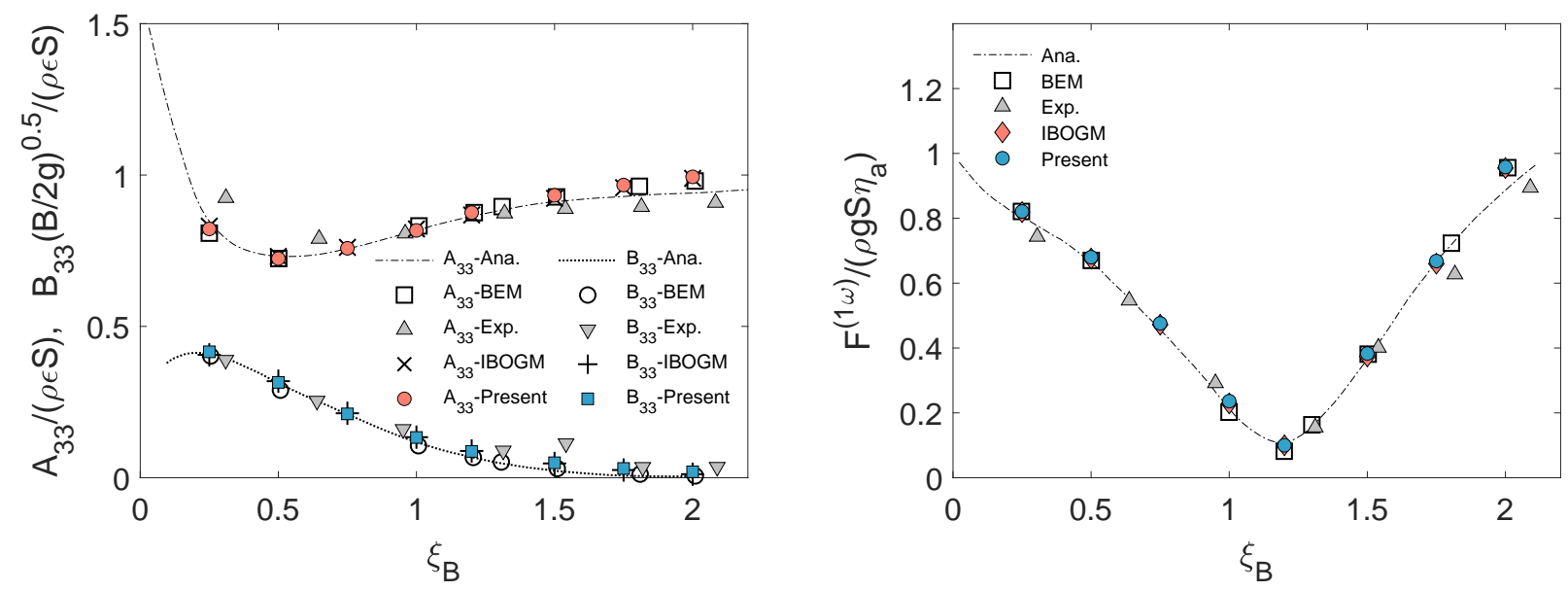

Figure 30: Heaving added mass and damping coefficients and normalized first-order Fourier forcecomponent for a Lewis-form barge. Here $S=B d$ is the mean submerged area of the barge. 


\section{Acknowledgment}

The first author (C. Tong) is funded by the Chinese Scholarship Council (No. 201906230317) and the Department of Mechanical Engineering at Technical University of Denmark. The fourth author is funded by the Centre for Autonomous Marine Operations and Systems (AMOS). The Research Council of Norway (Norges Forskningsråad) is acknowledged as the main sponsor of AMOS. This work was supported by the Research Council of Norway through the Centre of Excellence funding scheme, Project number 223254-AMOS. 


\section{${ }_{847}$ Data availability statement}

The data that supports the findings of this study are available in the supplementary material of this article.

\section{References}

[1] Chorin AJ. Numerical solution of the Navier-Stokes equations. Mathematics of computation. 1968;22(104):745-762.

[2] Taylor C, Hood P. A numerical solution of the Navier-Stokes equations using the finite element technique. Computers \& Fluids. 1973;1(1):73-100.

[3] Jacobsen NG, Fuhrman DR, Fredsøe J. A wave generation toolbox for the open-source CFD library: OpenFoamß. International Journal for numerical methods in fluids. 2012;70(9):10731088.

[4] Higuera P, Lara JL, Losada IJ. Realistic wave generation and active wave absorption for NavierStokes models: Application to OpenFOAM@. Coastal Engineering. 2013;71:102-118.

[5] Hu ZZ, Greaves D, Raby A. Numerical wave tank study of extreme waves and wave-structure interaction using OpenFoamB. Ocean Engineering. 2016;126:329-342.

[6] Bihs H, Kamath A, Chella MA, Aggarwal A, Arntsen ØA. A new level set numerical wave tank with improved density interpolation for complex wave hydrodynamics. Computers \& Fluids. 2016;140:191-208.

[7] Kamath A, Bihs H, Alagan Chella M, Arntsen ØA. Upstream-cylinder and downstream-cylinder influence on the hydrodynamics of a four-cylinder group. Journal of waterway, port, coastal, and ocean engineering. 2016;142(4):04016002.

[8] Oggiano L, Pierella F, Nygaard TA, De Vaal J, Arens E. Reproduction of steep long crested irregular waves with CFD using the VOF method. Energy Procedia. 2017;137:273-281.

[9] Kim SY, Kim KM, Park JC, Jeon GM, Chun HH. Numerical simulation of wave and current interaction with a fixed offshore substructure. International Journal of naval Architecture and ocean engineering. 2016;8(2):188-197.

[10] Tavelli M, Dumbser M. A staggered space-time discontinuous Galerkin method for the incompressible Navier-Stokes equations on two-dimensional triangular meshes. Computers \& Fluids. 2015;119:235-249.

[11] Fambri F, Dumbser M. Spectral semi-implicit and space-time discontinuous Galerkin methods for the incompressible Navier-Stokes equations on staggered Cartesian grids. Applied Numerical Mathematics. 2016;110:41-74.

[12] Fambri F, Dumbser M. Semi-implicit discontinuous Galerkin methods for the incompressible Navier-Stokes equations on adaptive staggered Cartesian grids. Computer Methods in Applied Mechanics and Engineering. 2017;324:170-203.

[13] Dumbser M, Fambri F, Furci I, Mazza M, Serra-Capizzano S, Tavelli M. Staggered discontinuous Galerkin methods for the incompressible Navier-Stokes equations: Spectral analysis and computational results. Numerical Linear Algebra with Applications. 2018;25(5):e2151.

[14] Dean RG, Dalrymple RA. Water wave mechanics for engineers and scientists. vol. 2. World Scientific Publishing Company; 1991. 
[15] Ramming HG, Kowalik Z. Numerical modelling of marine hydrodynamics. Elsevier; 1980.

[16] Faltinsen O. Sea loads on ships and offshore structures. vol. 1. Cambridge university press; 1993.

[17] Grilli ST, Skourup J, Svendsen IA. An efficient boundary element method for nonlinear water waves. Engineering Analysis with Boundary Elements. 1989;6(2):97-107.

[18] Grilli S. Fully nonlinear potential flow models used for long wave runup prediction. Chapter in Long-Wave Runup Models,(eds H Yeh, P Liu, and C Synolakis). 1997;p. 116-180.

[19] Ferrant P, Touzé DL, Pelletier K. Non-linear time-domain models for irregular wave diffraction about offshore structures. International journal for numerical methods in fluids. 2003;43(1011):1257-1277.

[20] Bai W, Teng B. Simulation of second-order wave interaction with fixed and floating structures in time domain. Ocean engineering. 2013;74:168-177.

[21] Harris JC, Dombre E, Benoit M, Grilli ST, et al. Fast integral equation methods for fully nonlinear water wave modeling. In: The Twenty-fourth International Ocean and Polar Engineering Conference. International Society of Offshore and Polar Engineers; 2014. .

[22] Bai W, Eatock Taylor R. Fully nonlinear simulation of wave interaction with fixed and floating flared structures. Ocean Engineering. 2009;36(3"):223 - 236.

[23] Ogilvie TF. Second-order hydrodynamic effects on ocean platforms. In: Proceedings of the International Workshop on Ship and Platform Motions, Berkeley; 1983. p. 205-265.

[24] Shao YL, Faltinsen OM. Towards efficient fully-nonlinear potential-flow solvers in marine hydrodynamics. In: ASME 2012 31st International Conference on Ocean, Offshore and Arctic Engineering. American Society of Mechanical Engineers Digital Collection; 2012. p. 369-380.

[25] Wang J, Faltinsen OM, Duan W. A high-order harmonic polynomial method for solving the Laplace equation with complex boundaries and its application to free-surface flows. Part I: two-dimensional cases. International Journal for Numerical Methods in Engineering. 2020;121(17):3893-3925.

[46] Rokhlin V. Rapid solution of integral equations of classical potential theory. Journal of computational physics. 1985;60(2):187-207.

[27] Fochesato C, Dias F. A fast method for nonlinear three-dimensional free-surface waves. Proceedings of the Royal Society A: Mathematical, Physical and Engineering Sciences. 2006;462(2073):2715-2735.

[28] Hague C, Swan C. A multiple flux boundary element method applied to the description of surface water waves. Journal of Computational Physics. 2009;228(14):5111-5128.

[29] Grilli S, Svendsen I. Corner problems and global accuracy in the boundary element solution of nonlinear wave flows. Engineering Analysis with Boundary Elements. 1990;7(4):178-195.

[30] Tanizawa K. The state of the art on numerical wave tank. In: Proceedings of 4th Osaka Colloquium on Seakeeping Performance of Ships 2000; 2000. p. 95-114.

[31] Li B, Fleming CA. A three dimensional multigrid model for fully nonlinear water waves. Coastal Engineering. 1997;30(3-4):235-258.

[32] Bingham HB, Zhang H. On the accuracy of finite-difference solutions for nonlinear water waves. Journal of Engineering Mathematics. 2007;58(1-4):211-228.

[33] Engsig-Karup AP, Bingham HB, Lindberg O. An efficient flexible-order model for 3D nonlinear water waves. Journal of computational physics. 2009;228(6):2100-2118. 
[34] Engsig-Karup AP, Madsen MG, Glimberg SL. A massively parallel GPU-accelerated model for analysis of fully nonlinear free surface waves. International Journal for Numerical Methods in Fluids. 2012;70(1):20-36.

[35] Ma Q, Wu G, Eatock Taylor R. Finite element simulation of fully non-linear interaction between vertical cylinders and steep waves. Part 1: methodology and numerical procedure. International Journal for Numerical Methods in Fluids. 2001;36(3):265-285.

[36] Wu G, Eatock Taylor R. The coupled finite element and boundary element analysis of nonlinear interactions between waves and bodies. Ocean Engineering. 2003;30(3):387-400.

[37] Wang C, Wu G. An unstructured-mesh-based finite element simulation of wave interactions with non-wall-sided bodies. Journal of fluids and structures. 2006;22(4):441-461.

[38] Yan S, Ma Q. Numerical simulation of fully nonlinear interaction between steep waves and 2D floating bodies using the QALE-FEM method. Journal of Computational physics. 2007;221(2):666-692.

[39] Deeks AJ, Cheng L. Potential flow around obstacles using the scaled boundary finite-element method. International journal for numerical methods in fluids. 2003;41(7):721-741.

[40] Wolf JP. The scaled boundary finite element method. John Wiley \& Sons; 2003.

[41] Li B, Cheng L, Deeks AJ. Wave diffraction by vertical cylinder using the scaled boundary finite element method. In: World Congress on Computational Mechanics VI. vol. 313. Beijing:[sn]; 2004. .

[42] Tao L, Song H, Chakrabarti S. Scaled boundary FEM solution of short-crested wave diffraction by a vertical cylinder. Computer methods in applied mechanics and engineering. 2007;197(14):232-242.

[43] Zietsman J. The coupled finite element and boundary integral analysis of ocean wave loading: A versatile tool. Computer methods in applied mechanics and engineering. 1984;44(2):153-176.

[44] Shao YL, Faltinsen OM. A harmonic polynomial cell (HPC) method for 3D Laplace equation with application in marine hydrodynamics. Journal of Computational Physics. 2014;274:312332.

[45] Ma S, Hanssen FC, Siddiqui MA, Greco M, Faltinsen OM. Local and global properties of the harmonic polynomial cell method: In-depth analysis in two dimensions. International Journal for Numerical Methods in Engineering. 2018;113(4):681-718.

[46] Bardazzi A, Lugni C, Antuono M, Graziani G, Faltinsen OM. Generalized HPC method for the Poisson equation. Journal of Computational Physics. 2015;299:630-648.

[47] Hanssen FCW. Non-Linear Wave-Body Interaction in Severe Waves. Norwegian University of Science and Technology; 2019.

[48] Fredriksen AG, Kristiansen T, Faltinsen OM. Experimental and numerical investigation of wave resonance in moonpools at low forward speed. Applied Ocean Research. 2014;47:28-46.

[49] Hanssen FCW, Greco M, Shao Y. The harmonic polynomial cell method for moving bodies immersed in a Cartesian background grid. In: ASME 2015 34th International Conference on Ocean, Offshore and Arctic Engineering. American Society of Mechanical Engineers Digital Collection; 2015. .

[50] Zhu W, Greco M, Shao Y. Improved HPC method for nonlinear wave tank. International Journal of Naval Architecture and Ocean Engineering. 2017;9(6):598-612.

[51] Hanssen FC, Bardazzi A, Lugni C, Greco M. Free-surface tracking in 2D with the harmonic polynomial cell method: Two alternative strategies. International Journal for Numerical Methods 
in Engineering. 2018;113(2):311-351.

[52] Robaux F, Benoit M. Development and validation of a numerical wave tank based on the Harmonic Polynomial Cell and Immersed Boundary methods to model nonlinear wave-structure interaction. arXiv preprint arXiv:200908937. 2020;.

[53] Liang H, Santo H, Shao Y, Law YZ, Chan ES. Liquid sloshing in an upright circular tank under periodic and transient excitations. Physical Review Fluids. 2020;5(8):084801.

[54] Tong C, Shao Y, Hanssen FCW, Li Y, Xie B, Lin Z. Numerical analysis on the generation, propagation and interaction of solitary waves by a Harmonic Polynomial Cell Method. Wave Motion. 2019;88:34-56.

[55] Shen Y, Marilena G, Faltinsen OM, Ma S. Numerical study towards closed fish farms in waves using two Harmonic Polynomial Cell methods. In: Proceedings of 35th International Workshop on Water Waves and Floating Bodies. The International Workshop on Water Waves and Floating Bodies; 2020. .

[56] Kontos S. Robust Numerical Methods for Nonlinear Wave-Structure Interaction in a Moving Frame of Reference. Technical University of Denmark; 2016.

[57] Liu Y, Xue M, Yue DK. Computations of fully nonlinear three-dimensional wave-wave and wavebody interactions. Part 2. Nonlinear waves and forces on a body. Journal of Fluid Mechanics. 2001;438:41.

[58] Tanizawa K. A nonlinear simulation method of 3-D body motions in waves (1st Report). Journal of the Society of Naval Architects of Japan. 1995;1995(178):179-191.

[59] Wu G. Transient motion of floating body in steep water waves. In: Proc. 11th Workshop on water waves and Floating Bodies; 1996. .

[60] Beck R. Nonlinear ship motion computations using the desingularized method. In: Proc. 20th Symp. on Naval Hydrodynamics; 1996. p. 227-246.

[61] Kashiwagi M. Non-linear simulations of wave-induced motions of a floating body by means of the mixed Eulerian-Lagrangian method. Proceedings of the Institution of Mechanical Engineers, Part C: Journal of Mechanical Engineering Science. 2000;214(6):841-855.

[62] Wu G, Hu Z. Simulation of nonlinear interactions between waves and floating bodies through a finite-element-based numerical tank. Proceedings of the Royal Society of London Series A: Mathematical, Physical and Engineering Sciences. 2004;460(2050):2797-2817.

[63] Bandyk PJ, Beck RF. The acceleration potential in fluid-body interaction problems. Journal of Engineering Mathematics. 2011;70(1-3):147-163.

[64] Zhao R, Faltinsen O, Aarsnes J. Water entry of arbitrary two-dimensional sections with and without flow separation. In: Proceedings of the 21st symposium on naval hydrodynamics; 1996. p. $408-423$.

[65] Greco M. A Two-dimensional Study of Green-water Loading (Ph. D. dissertation). Norwegian University of Science and Technology, Trondheim, Norwegian; 2001.

[66] Sun H. A boundary element method applied to strongly nonlinear wave-body interaction problems. Norwegian University of Science and Technology; 2007.

[67] Newman JN. Marine hydrodynamics. The MIT Press; 2017.

[68] Rienecker M, Fenton J. A Fourier approximation method for steady water waves. Journal of fluid mechanics. 1981;104:119-137.

[69] Fenton J. The numerical solution of steady water wave problems. Computers \& Geosciences. 
1036
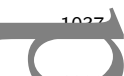
of Computational Physics. 2014;257:863-883.

82] Tasai F, Koterayama W. Nonlinear hydrodynamic forces acting on cylinders heaving on the surface of a fluid. Rep Res Inst Appl Mech Kyushu Univ. 1976;24:1-39.

[83] Grytøyr G. A higher-order boundary element method and applications to marine hydrodynamics. Norwegian University of Science and Technology, Trondheim, Norwegian; 2008.

[84] Wang J, Faltinsen OM. A harmonic polynomial method based on Cartesian grids with local refinement for complex wave-body interactions. In: Proceedings of 33rd International Workshop on Water Waves and Floating Bodies. The International Workshop on Water Waves and Floating Bodies; 2018. . 